\title{
Feasibility of motion laws for planar one degree of freedom linkage mechanisms at dead point configurations
}

\author{
E. Lores García*, J. M. Veciana Fontanet and L. Jordi Nebot \\ Department of Mechanical Engineering, Universitat Politècnica de Catalunya, Diagonal 647, \\ Barcelona, Spain, 08028 \\ eduard.lores@estudiant.upc.edu,joaquim.maria.veciana@upc.edu, lluisa.jordi@upc.edu \\ *-Corresponding author
}

\begin{abstract}
This paper proposes an analytical solution of the Inverse Kinematics (IK) problem at dead point configurations for any planar one degree of freedom linkage mechanism, with regard to the continuity $\mathcal{C}^{n}$ of the motion law. The systems analyzed are those whose elements are linked with lower pairs and do not present redundancies. The study aims to provide the user with some rules to facilitate the design of feasible motion profiles to be reproduced by conventional electrical actuators at these configurations. During the last decades, several methods and techniques have been developed to study this specific configuration. However, these techniques are mainly focused on solving numerically the IK indeterminacy, rather than analyzing the motion laws that the mechanisms are able to perform at these particular configurations. The analysis presented in this paper has been carried out differentiating and applying l'Hôpital's rule to the system of constraint equations $\boldsymbol{\phi}(\boldsymbol{q})$ of the mechanism. The study also considers the feasibility of the timedomain profiles to be reproduced with conventional electrical actuators (i.e. AC/DC motors, linear actuators, etc.). To show the usefulness and effectiveness of the method, the development includes the analytical application and numerical simulations for two common one degree of freedom systems: a slider-crank and a four linkage mechanisms. Finally, experimental results are presented on a four linkage mechanism test bed.
\end{abstract}

Keywords: inverse kinematics, linkage mechanism, singular configuration, dead point, motion law.

\section{Introduction}

The design of motion profiles of machine elements has been widely studied in research areas such as mechanical engineering and control engineering. Commonly, the transmission chain between the actuator coordinate, commanded by the user, and the coordinate that describes the desired motion, is linear (pulley belt [1], gear chain [2], rack and pinion, etc.) (Figure 1). In this case, the constraints and limitations to perform any profile generally come from the actuator or control performances. Nevertheless, when the transmission chain is a linkage mechanism, it is necessary to deal with additional constraints, such as the singularities of the targeted coordinate within its functional range [3]. The actuator coordinate, however, has typically no singularities.

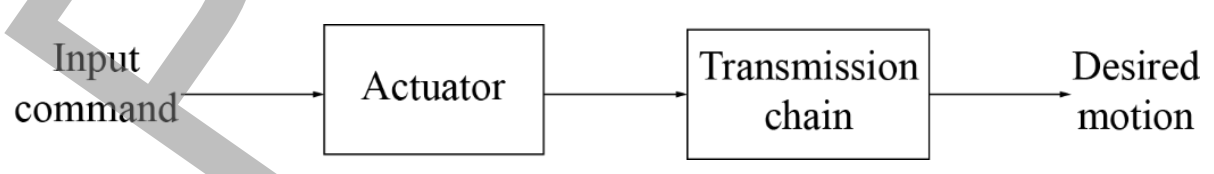

Figure 1. Block diagram of the system. 
In the study of kinematics in linkage mechanisms with planar motion, the mobility can be described by a set of generalized coordinates and generalized velocities. The coordinates to describe the configuration of the mechanism are named generalized coordinates. The minimum number of them to fully define the state of the mechanism are the so-called independent coordinates, while the rest are known as dependent coordinates. In the case of a planar one degree of freedom mechanism, there is only one independent coordinate. Generically, the aim of the user is to design a time domain evolution of this coordinate, described by means of a motion law or profile. Thus, the inverse kinematics (IK) approach could be used to calculate the actuator command. However, if this coordinate has dead point configurations within its accessible configuration range, IK leads to algebraic indeterminacies. This paper addresses which type of time-domain profiles can be performed, by the independent coordinate, in presence of dead points. The analysis is carried out with regard to the continuity $\mathcal{C}^{n}$ of the profile and its feasibility to be reproduced with conventional electrical actuators (i.e. AC/DC motors, linear actuators, etc.).

In order to find a solution to IK near dead points, Whitney [4] suggested using the pseudoinverse of the Jacobian matrix $(J)$. The main drawback of this method is that it has stability problems in the neighborhood of dead points. Balestrino et al. [5] and Wolovich and Elliot [6] proposed to use the transpose of $J$ instead of its inverse which, according to Buss [7], is a good approximation when scaled by some small scalar $\alpha$. Another approach is the well-known damped least-squares method (DLS) introduced by Nakamura and Hanafusa [8] and Wampler [9]. This technique uses a damping factor $k$ to ensure that an inverse kinematic solution exists in the vicinity of a dead point by allowing the independent coordinate to deviate from the reference trajectory. There have been several authors that presented methods to find an appropriate value for $k$ ([8], [10], [11], [12], [13], [14], [15]). Similar methods to the DLS are exposed by Xiang et al. [16] and Sugihara [17].

Another approach to solve the IK problem is based on a null-space path tracking technique presented by Nenchev [18], and Nenchev and Uchiyama [19]. This method, based on the work of Kieffer [20] and known as Singularity Consistent (SC) method, proposes to reparametrize the path of the independent coordinate in the neighborhood of a dead point. The main idea is to parametrize the independent coordinate trajectory with a smooth function $g(\alpha)$, where $\alpha$ is not time and is treated as a dependent coordinate. Nenchev et al. [21] analytically demonstrated the equivalence between the Singularity Consistent and the Jacobian adjoint matrix technique used by Tchoń and Dulęba [20] and Senft and Hirzinger [23].

Other methods for solving the IK problem are based on artificial neural networks ([24], [25]). Aristidou and Lasenby [26] suggested a heuristic method called Forward And Backward Reaching Inverse Kinematics (FABRIK) that takes the last calculated position of the dependent coordinates to find the future values in a forward and backward iterative mode. The Feedback Inverse Kinematics (FIK) presented by Pechev [27] uses a feedback loop to minimize the difference between the actual and the target velocity. Vargas [28] introduced the Filtered Inverse algorithm (FI), which dynamically estimates the inverse of the Jacobian matrix $J$. 
The present paper proposes an analytical solution of the IK problem at dead point configurations, for any planar one degree of freedom linkage mechanism, with regard to the continuity $\mathcal{C}^{n}$ of a motion law that describes the time-evolution of the independent coordinate. The systems analyzed are those whose elements are linked with lower pairs and do not present redundancies. The novelty of this study is to provide the user with some rules to facilitate the design of feasible motion profiles of the independent coordinate at these configurations, while dealing with the limitations of conventional electrical actuators. The main contributions of this development are: i) The study of the feasibility of the time-domain profiles, with regard to the continuity $\mathcal{C}^{n}$, to be reproduced with the above-mentioned actuators; $i$ ) The analysis carried out by differentiating and applying l'Hôpital's rule to the system of constraint equations $\phi(q)$ of the mechanism, which is a different approach from previous work; iii) The specific rules to design motion profiles around a dead point configuration, taking into consideration the limitations of these actuators and all the casuistry involved, related with the values of the derivatives of the independent coordinate, and $i v$ ) the verification of the conclusions obtained through simulation and experimental results.

The paper is organized as follows. Section 2 introduces the problem formulation of the IK approach in the neighborhood of a dead point. Section 3 analyzes the limitations of a conventional electrical actuator to perform a command input with regard to the continuity $\mathcal{C}^{n}$. Section 4 proposes the workspace solutions of the problem stated in section 2 and particularizes the results for a slider-crank mechanism. Some simulations to confirm the goodness of the development are carried out in section 5 and experimental results are presented in section 6 . Finally, conclusions are drawn in section 7.

\section{Problem formulation}

The mobility of a mechanism of $m$ degrees of freedom can be described by a set of $n+m$ generalized coordinates $q=\left\{q_{1}, q_{2}, \ldots, q_{n}, q_{n+1}, \ldots, q_{n+m}\right\}^{\mathrm{T}}$ and generalized velocities $\dot{\boldsymbol{q}}=$ $\left\{\dot{q}_{1}, \dot{q}_{2}, \ldots, \dot{q}_{n}, \dot{q}_{n+1}, \ldots, \dot{q}_{n+m}\right\}^{\mathrm{T}}$. The set of geometric variables $\boldsymbol{q}$ defines all the possible configurations of the mechanism, while its derivatives $\dot{\boldsymbol{q}}$ and $\ddot{\boldsymbol{q}}$ define the distribution of the mechanism velocities and accelerations. The minimum set of $m$ coordinates to fully define the configuration of the mechanism are called independent coordinates $\left(\boldsymbol{q}^{\mathrm{i}}=\left\{q_{n+1}, \ldots, q_{n+m}\right\}^{\mathrm{T}}\right)$, while the remaining $n$ are known as dependent coordinates $\left(\boldsymbol{q}^{\mathrm{d}}=\left\{q_{1}, q_{2}, \ldots, q_{n}\right\}^{\mathrm{T}}\right)$. In the case of a planar one degree of freedom mechanism $(m=1)$, there is only one independent coordinate $q^{\mathrm{i}}$. Generically, this coordinate is used, by means of a motion law in the time domain, to describe the movement of the mechanism. If the actuator is commanded through a different coordinate than the independent one, the IK technique could be used to calculate the actuator command. Nevertheless, the IK approach in mechanisms presents problems when the independent coordinate is found at a dead point within its accessible configuration's range. Actually, this problem can be summarized as an algebraic indeterminacy. An example of a planar one degree of freedom mechanism with dead points is the four linkage mechanism presented in Figure 2 a), where $\boldsymbol{q}=\left\{q_{1}, q_{2}, q_{3}\right\}^{\mathrm{T}}$. In this case, the generalized coordinate $q_{3}$ presents a dead point when $q_{1}=q_{2}($ Figure $2 \mathrm{~b})$ ). 


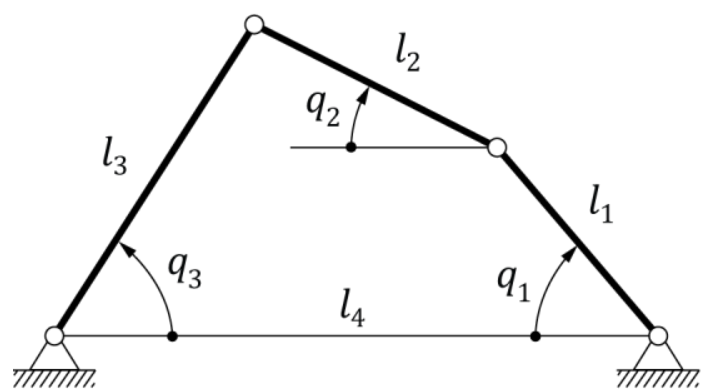

a)

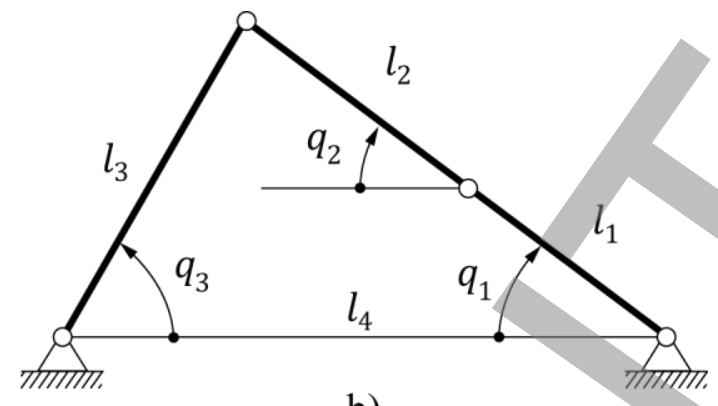

b)

Figure 2. a) Four linkage mechanism with 3 generalized coordinates $\left(q_{1}, q_{2}\right.$ and $\left.q_{3}\right)$; b) Dead point configuration for $q_{3}$.

In a planar one degree of freedom mechanism, in general, the relation between the $n+1$ generalized coordinates can be obtained from the constraint equations $\phi_{i}(\boldsymbol{q})$ of the mechanism.

$$
\boldsymbol{\phi}(\boldsymbol{q})=\left\{\begin{array}{c}
\phi_{1}(\boldsymbol{q}) \\
\vdots \\
\phi_{n}(\boldsymbol{q})
\end{array}\right\}=0
$$

According to Cardona and Clos [29], Eq. (1) is differentiated in order to calculate the velocities $\dot{\boldsymbol{q}}$ of the mechanism

$$
\phi_{q} \dot{q}=0
$$

where $\boldsymbol{\phi}_{q}$ is known as the Jacobian matrix of the system of equations and is calculated according to Eq. (3)

$$
\phi_{q}=\left[\begin{array}{ccc}
\frac{\partial \phi_{1}(\boldsymbol{q})}{\partial q_{1}} & \cdots & \frac{\partial \phi_{1}(\boldsymbol{q})}{\partial q_{n+1}} \\
\vdots & & \vdots \\
\frac{\partial \phi_{n}(\boldsymbol{q})}{\partial q_{1}} & \cdots & \frac{\partial \phi_{n}(\boldsymbol{q})}{\partial q_{n+1}}
\end{array}\right]
$$

Splitting the dependent and independent terms in Eq. (2)

$$
\begin{gathered}
\boldsymbol{\phi}_{q}^{\mathrm{d}} \dot{\boldsymbol{q}}^{\mathrm{d}}+\boldsymbol{\phi}_{q}^{\mathrm{i}} \dot{q}^{\mathrm{i}}=0 \\
\dot{\boldsymbol{q}}^{\mathrm{d}}=-\boldsymbol{\phi}_{q}^{\mathrm{d}^{-1}} \boldsymbol{\phi}_{q}^{\mathrm{i}} \dot{q}^{\mathrm{i}}=-\frac{\operatorname{adj}\left(\boldsymbol{\phi}_{q}^{\mathrm{d}}\right)}{\operatorname{det}\left(\boldsymbol{\phi}_{q}^{\mathrm{d}}\right)} \boldsymbol{\phi}_{q}^{\mathrm{i}} \dot{q}^{\mathrm{i}}
\end{gathered}
$$

with 


$$
\begin{gathered}
\boldsymbol{\phi}_{q}^{\mathrm{d}}=\left[\begin{array}{ccc}
\frac{\partial \phi_{1}(\boldsymbol{q})}{\partial q_{1}} & \cdots & \frac{\partial \phi_{1}(\boldsymbol{q})}{\partial q_{n}} \\
\vdots & & \vdots \\
\frac{\partial \phi_{n}(\boldsymbol{q})}{\partial q_{1}} & \cdots & \frac{\partial \phi_{n}(\boldsymbol{q})}{\partial q_{n}}
\end{array}\right] \\
\boldsymbol{\phi}_{q}^{\mathrm{i}}=\left\{\begin{array}{c}
\frac{\partial \phi_{1}(\boldsymbol{q})}{\partial q^{\mathrm{i}}} \\
\vdots \\
\frac{\partial \phi_{n}(\boldsymbol{q})}{\partial q^{\mathrm{i}}}
\end{array}\right\}
\end{gathered}
$$

When a mechanism is found at a dead point configuration for the generalized independent coordinate $q^{\mathrm{i}}$, its corresponding velocity $\dot{q}^{\mathrm{i}}$ has a null value regardless the motion of the rest of the mechanism. Therefore, to have a solution for the generalized dependent coordinates $\dot{\boldsymbol{q}}^{\mathrm{d}}$ different than zero, the value of the determinant of $\phi_{q}^{d}$ must also be null. As a consequence, the value of the velocities $\dot{\boldsymbol{q}}^{\mathrm{d}}$ cannot be numerically defined using Eq. (5), because of this indeterminacy.

In the case of the mechanism shown in Figure 2, the relation between $q_{1}, q_{2}$ and $q_{3}$ can be obtained from the constraint equations $\phi_{i}(\boldsymbol{q})$ of the mechanism.

$$
\boldsymbol{\phi}(\boldsymbol{q})=\boldsymbol{\phi}\left(q_{1}, q_{2}, q_{3}\right)=\left\{\begin{array}{l}
\phi_{1}\left(q_{1}, q_{2}, q_{3}\right) \\
\phi_{2}\left(q_{1}, q_{2}, q_{3}\right)
\end{array}\right\}=0
$$

And the Jacobian matrix of the system of equations will be

$$
\boldsymbol{\phi}_{q}=\left[\begin{array}{lll}
\frac{\partial \phi_{1}\left(q_{1}, q_{2}, q_{3}\right)}{\partial q_{1}} & \frac{\partial \phi_{1}\left(q_{1}, q_{2}, q_{3}\right)}{\partial q_{2}} & \frac{\partial \phi_{1}\left(q_{1}, q_{2}, q_{3}\right)}{\partial q_{3}} \\
\frac{\partial \phi_{2}\left(q_{1}, q_{2}, q_{3}\right)}{\partial q_{1}} & \frac{\partial \phi_{2}\left(q_{1}, q_{2}, q_{3}\right)}{\partial q_{2}} & \frac{\partial \phi_{2}\left(q_{1}, q_{2}, q_{3}\right)}{\partial q_{3}}
\end{array}\right]
$$

If $q_{3}$ is considered the independent coordinate $q^{\mathrm{i}}$, and $q_{1}$ and $q_{2}$ are treated as dependent coordinates $\boldsymbol{q}^{\text {d }}$, Eq. (4) can be rewritten as

$$
\boldsymbol{\phi}_{q}^{\mathrm{d}}\left\{\dot{q}_{1}, \dot{q}_{2}\right\}^{\mathrm{T}}+\boldsymbol{\phi}_{q}^{\mathrm{i}} \dot{q}_{3}=0
$$

Then,

$$
\left\{\begin{array}{l}
\dot{q}_{1} \\
\dot{q}_{2}
\end{array}\right\}=-\frac{\operatorname{adj}\left(\boldsymbol{\phi}_{q}^{\mathrm{d}}\right)}{\operatorname{det}\left(\boldsymbol{\phi}_{q}^{\mathrm{d}}\right)} \boldsymbol{\phi}_{q}^{\mathrm{i}} \dot{q}_{3}
$$


Regarding the acceleration vector $\ddot{\boldsymbol{q}}^{\mathrm{d}}$, a similar development can be obtained by differentiating Eq. (2)

$$
\dot{\boldsymbol{\phi}}_{q} \dot{\boldsymbol{q}}+\boldsymbol{\phi}_{q} \ddot{\boldsymbol{q}}=0
$$

Splitting again the dependent and independent terms of Eq. (12)

$$
\begin{gathered}
\dot{\boldsymbol{\phi}}_{q} \dot{\boldsymbol{q}}+\boldsymbol{\phi}_{q}^{\mathrm{d}} \ddot{\boldsymbol{q}}^{\mathrm{d}}+\boldsymbol{\phi}_{q}^{\mathrm{i}} \ddot{q}^{\mathrm{i}}=0 \\
\ddot{\boldsymbol{q}}^{\mathrm{d}}=-\boldsymbol{\phi}_{q}^{\mathrm{d}^{-1}}\left[\boldsymbol{\phi}_{q}^{\mathrm{i}} \ddot{q}^{\mathrm{i}}+\dot{\boldsymbol{\phi}}_{q} \dot{\boldsymbol{q}}\right]=-\frac{\operatorname{adj}\left(\boldsymbol{\phi}_{q}^{\mathrm{d}}\right)}{\operatorname{det}\left(\boldsymbol{\phi}_{q}^{\mathrm{d}}\right)}\left[\boldsymbol{\phi}_{q}^{\mathrm{i}} \ddot{q}^{\mathrm{i}}+\dot{\boldsymbol{\phi}}_{q} \dot{\boldsymbol{q}}\right]
\end{gathered}
$$

with

$$
\dot{\boldsymbol{\phi}}_{q}=\frac{\mathrm{d}}{\mathrm{d} t}\left\{\boldsymbol{\phi}_{q}\right\}=\left[\begin{array}{ccc}
\frac{\mathrm{d}}{\mathrm{d} t}\left\{\frac{\partial \phi_{1}(\boldsymbol{q})}{\partial q_{1}}\right\} & \cdots & \frac{\mathrm{d}}{\mathrm{d} t}\left\{\frac{\partial \phi_{1}(\boldsymbol{q})}{\partial q_{n+1}}\right\} \\
\vdots & & \vdots \\
\frac{\mathrm{d}}{\mathrm{d} t}\left\{\frac{\partial \phi_{n}(\boldsymbol{q})}{\partial q_{1}}\right\} & \cdots & \frac{\mathrm{d}}{\mathrm{d} t}\left\{\frac{\partial \phi_{n}(\boldsymbol{q})}{\partial q_{n+1}}\right\}
\end{array}\right]
$$

When the independent coordinate $q^{\mathrm{i}}$ is located in one of its dead point configurations, the value of the numerator of Eq. (14) is also null (see Appendix A). Thus, analogously to the case of the velocities $\dot{\boldsymbol{q}}^{\mathrm{d}}$, the algebraic indeterminacy presented in Eq. (14) does not allow the calculation of the accelerations $\ddot{\boldsymbol{q}}^{\mathrm{d}}$. Therefore, Eqs. (5) and (14) seem to imply that the motion law of the independent coordinate $q^{\mathrm{i}}$ cannot be used to track the kinematics of the entire mechanism in this specific configuration.

In the four linkage mechanism presented in Figure 2, the accelerations $\left\{\ddot{q}_{1}, \ddot{q}_{2}\right\}^{\mathrm{T}}$ are

$$
\left\{\begin{array}{c}
\ddot{q}_{1} \\
\ddot{q}_{2}
\end{array}\right\}=-\frac{\operatorname{adj}\left(\phi_{q}^{\mathrm{d}}\right)}{\operatorname{det}\left(\boldsymbol{\phi}_{q}^{\mathrm{d}}\right)}\left[\boldsymbol{\phi}_{q}^{\mathrm{i}} \ddot{q}_{3}+\dot{\boldsymbol{\phi}}_{q}\left\{\dot{q}_{1}, \dot{q}_{2}, \dot{q}_{3}\right\}^{\mathrm{T}}\right]
$$

The aim of this study is to prove which is the minimum continuity $\mathcal{C}^{n}$ required by the motion law of the independent coordinate in presence of dead points in order to be achievable by a conventional electrical actuator while controlling a dependent coordinate. 


\section{Capability of conventional actuators}

In this section it is defined the minimum continuity $\mathcal{C}^{n}$ that an input command must have in order to be performed with a reasonable accuracy by a conventional actuator. To this purpose, the limitations caused by the discontinuities in position, velocity and acceleration are considered. However, a quantitative analysis of the capability of the actuators is not included. This means that the frequency range in which the actuators are able to respond is not considered. As an example, the input command of the generalized coordinate $q_{1}$ of the four linkage mechanism presented in Figure 2 is studied.

In conventional electrical actuators such as AC or DC servomotors, the electrical time constant and the magnetic diffusion time are about few milliseconds (see [30]). Therefore, a constant stationary torque and, consequently, a constant stationary acceleration are fully available after this transition time $t_{\mathrm{t}}$ when a step is used as an electrical input command.

Assume that the coordinate $q_{1}$ follows the time profile showed in Figure 3 a):

$$
\begin{aligned}
& q_{1}(t)=\left\{\begin{array}{cl}
0 & -\infty<t \leq t_{0^{-}} \\
b_{1} t & t_{0^{+}} \leq t<+\infty
\end{array} \quad \dot{q}_{1}(t)=\left\{\begin{array}{cc}
0 & -\infty<t \leq t_{0^{-}} \\
b_{1} & t_{0^{+}} \leq t<+\infty
\end{array}\right.\right. \\
& \ddot{q}_{1}(t)=\left\{\begin{array}{cc}
0 & -\infty<t \leq t_{0^{-}} \\
b_{1} \delta(t) & t_{0^{-}}<t<t_{0^{+}} \\
0 & t_{0^{+}} \leq t<+\infty
\end{array}\right.
\end{aligned}
$$

where $t_{0^{-}}$and $t_{0^{+}}$are the instant of time just before and after of the starting point of the motion profile, respectively. The parameter $b_{1}$ refers to the coefficient of the generic polynomial motion law of $q_{1}(t)$. This type of movement is $\mathcal{C}^{0}$ and imply an impulse of acceleration (Eq. (17)). Although this motion can physically be approximated by a percussive torque, the majority of the conventional actuators cannot execute it.

Consider now the $\mathcal{C}^{1}$ input command presented in Figure $3 \mathrm{~b}$ ), where $b_{2}$ is a generic coefficient of the new polynomial expression of $q_{1}(t)$ :

$$
\begin{gathered}
q_{1}(t)=\left\{\begin{array}{ccc}
0 & -\infty<t \leq t_{0^{-}} & \dot{q}_{1}(t)=\left\{\begin{array}{cc}
0 & -\infty<t \leq t_{0^{-}} \\
2 b_{2} t & t_{0^{+}} \leq t<+\infty
\end{array}\right. \\
b_{0^{+}} \leq t<+\infty
\end{array}\right. \\
\ddot{q}_{1}(t)=\left\{\begin{array}{cc}
0 & -\infty<t \leq t_{0^{-}} \\
2 b_{2} & t_{0^{+}} \leq t<+\infty
\end{array}\right.
\end{gathered}
$$

Unlike the case detailed in Eq. (17), this acceleration profile can be performed with more or less precision with a certain delay of $t_{\mathrm{t}}$ milliseconds, as exposed above (real acceleration drawn in Figure 3 is just for reference). Its accuracy will depend on the frequency range where the 
actuators are able to respond. Thus, the hypothesis assumed in this study is that any input command performed by conventional electrical actuators must be, at least, $\mathcal{C}^{1}$.
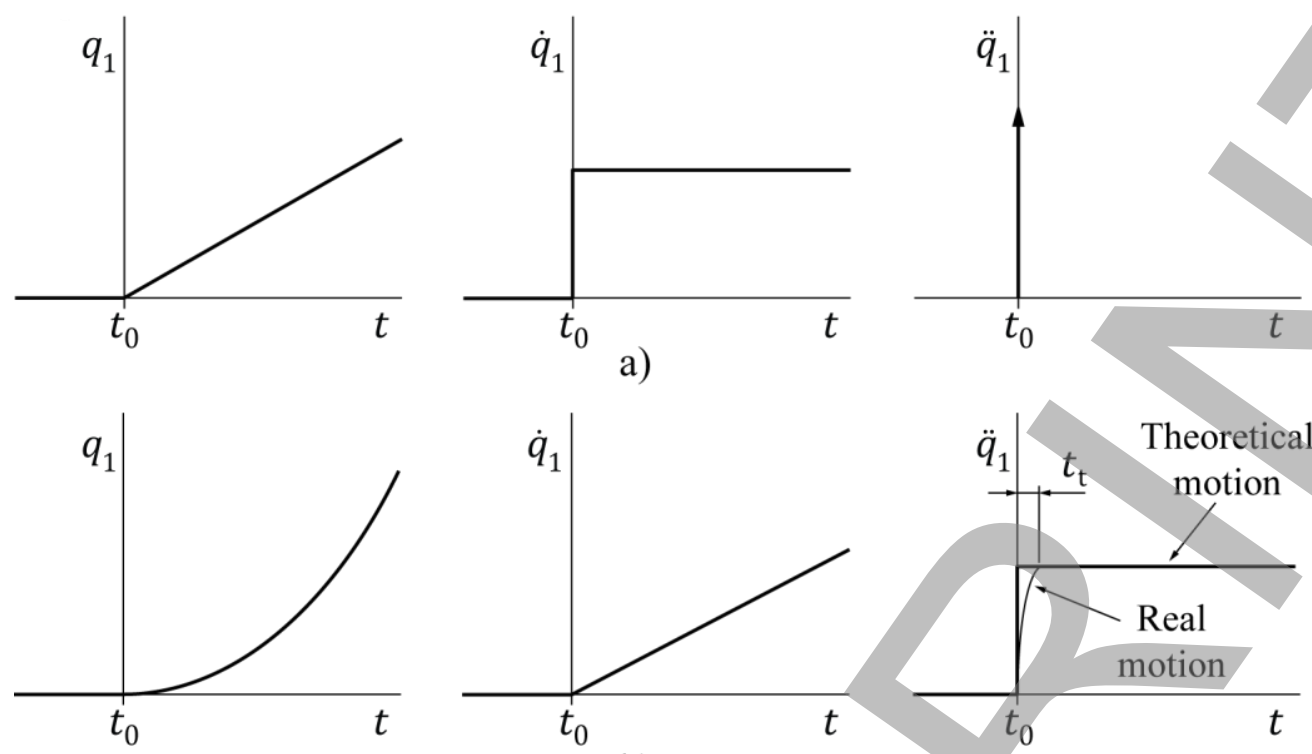

b)

Figure 3: Input command with a discontinuity in the velocity $\dot{q}_{1}$ a) and acceleration $\ddot{q}_{1}$ b).

\section{Kinematic study at dead point configurations for 1 degree of freedom linkage mechanisms}

Assuming a one degree of freedom mechanism, the purpose of this section is to analyze the continuity $\mathcal{C}^{n}$ of the independent coordinate motion law, when dead points are present, by solving the algebraic indeterminacy shown in section 2 . This study contemplates the velocity and acceleration analysis of the dependent coordinates based on the motion profile described by the time evolution of the independent coordinate. For a better understanding while developing the generic solution, all the results are particularized to the slider-crank mechanism presented in Figure 4 a) when found at the dead point configuration of the independent coordinate $q_{3}$ shown in Figure 4 b). The conclusions obtained in this study are valid for any planar one degree of freedom mechanism, with lower pairs and without redundancies, when the independent coordinate is found at a dead point.

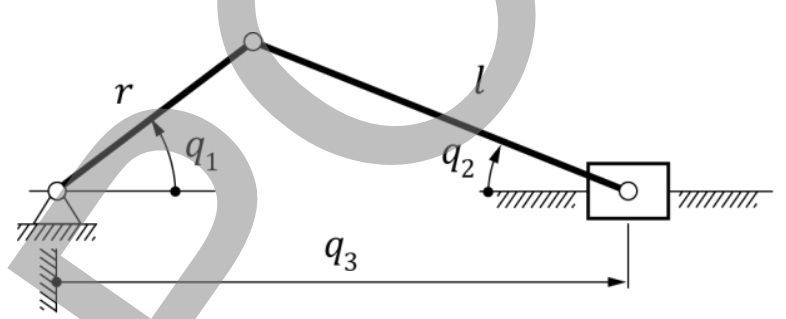

a)

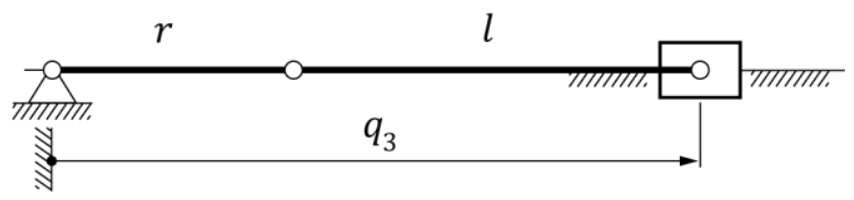

b)

Figure 4: Slider crank mechanism in a general configuration a) and at a dead point of the coordinate $q_{3}$ b). 


\section{$\underline{4.1 \text { Velocity analysis }}$}

As stated in Eq. (5), the $n$ velocities $\dot{\boldsymbol{q}}^{\mathrm{d}}$ of the dependent coordinates are proportional to the velocity $\dot{q}^{\mathrm{i}}$ of the independent coordinate. At a dead point configuration, the generalized velocity $\dot{q}^{\mathrm{i}}$ has a null value regardless the motion of the rest of the mechanism. In order to have a solution for $\dot{\boldsymbol{q}}^{\mathrm{d}}$ different than zero, the determinant of the matrix $\boldsymbol{\phi}_{q}^{\mathrm{d}}$ must also be null. This fact implies an algebraic indeterminacy $0 / 0$ in the calculation of $\dot{\boldsymbol{q}}^{\mathrm{d}}$

$$
\left.\dot{\boldsymbol{q}}^{\mathrm{d}}\right|_{\text {d.p. }}=\lim _{q^{\mathrm{i}} \rightarrow \text { d.p. }}\left(-\frac{\operatorname{adj}\left(\boldsymbol{\phi}_{q}^{\mathrm{d}}\right)}{\operatorname{det}\left(\boldsymbol{\phi}_{q}^{\mathrm{d}}\right)} \boldsymbol{\phi}_{q}^{\mathrm{i}} \dot{q}^{\mathrm{i}}\right)=\frac{0}{0}
$$

The indeterminacy shown in Eq. (19) can be solved by applying L'Hôpital's rule and imposing $\dot{q}^{\mathrm{i}}=0$

$$
\begin{aligned}
& \left.\dot{\boldsymbol{q}}^{\mathrm{d}}\right|_{\text {d.p. }}=\lim _{q^{\mathrm{i} \rightarrow \text { d.p. }}}\left(-\frac{\frac{\mathrm{d}}{\mathrm{d} t}\left(\operatorname{adj}\left(\boldsymbol{\phi}_{q}^{\mathrm{d}}\right) \boldsymbol{\phi}_{q}^{\mathrm{i}} \dot{q}^{\mathrm{i}}\right)}{\frac{\mathrm{d}}{\mathrm{d} t}\left(\operatorname{det}\left(\boldsymbol{\phi}_{q}^{\mathrm{d}}\right)\right)}\right)= \\
& =\lim _{q^{\mathrm{i}} \rightarrow \text { d.p. }}\left(-\frac{\frac{\mathrm{d}}{\mathrm{d} t}\left(\operatorname{adj}\left(\boldsymbol{\phi}_{q}^{\mathrm{d}}\right)\right) \boldsymbol{\phi}_{q}^{\mathrm{i}} \dot{q}^{\mathrm{i}}+\operatorname{adj}\left(\boldsymbol{\phi}_{q}^{\mathrm{d}}\right)\left\{\dot{\boldsymbol{\phi}}_{q}^{\mathrm{i}} \dot{q}^{\mathrm{i}}+\boldsymbol{\phi}_{q}^{\mathrm{i}} \ddot{q}^{\mathrm{i}}\right\}}{\frac{\partial}{\partial \boldsymbol{q}}\left(\operatorname{det}\left(\boldsymbol{\phi}_{q}^{\mathrm{d}}\right)\right) \dot{\boldsymbol{q}}}\right)= \\
& =\lim _{q^{i} \rightarrow \text { d.p. }}\left(-\frac{\frac{\mathrm{d}}{\mathrm{d} t}\left(\operatorname{adj}\left(\boldsymbol{\phi}_{q}^{\mathrm{d}}\right)\right) \boldsymbol{\phi}_{q}^{\mathrm{i}} \dot{q}^{\mathrm{i}}+\operatorname{adj}\left(\boldsymbol{\phi}_{q}^{\mathrm{d}}\right)\left\{\dot{\boldsymbol{\phi}}_{q}^{\mathrm{i}} \dot{q}^{\mathrm{i}}+\boldsymbol{\phi}_{q}^{\mathrm{i}} \ddot{q}^{\mathrm{i}}\right\}}{\left\{\frac{\partial}{\partial \boldsymbol{q}^{\mathrm{d}}}\left(\operatorname{det}\left(\boldsymbol{\phi}_{q}^{\mathrm{d}}\right)\right), \frac{\partial}{\partial q^{\mathrm{i}}}\left(\operatorname{det}\left(\boldsymbol{\phi}_{q}^{\mathrm{d}}\right)\right)\right\}\left\{\dot{\boldsymbol{q}}^{\mathrm{d}}, \dot{q}^{\mathrm{i}}\right\}^{\mathrm{T}}}\right)= \\
& =-\left.\frac{\operatorname{adj}\left(\phi_{q}^{\mathrm{d}}\right) \boldsymbol{\phi}_{q}^{\mathrm{i}} \ddot{q}^{\mathrm{i}}}{\frac{\partial}{\partial \boldsymbol{q}^{\mathrm{d}}}\left(\operatorname{det}\left(\boldsymbol{\phi}_{q}^{\mathrm{d}}\right)\right) \dot{\boldsymbol{q}}^{\mathrm{d}}}\right|_{\text {d.p. }}
\end{aligned}
$$

In order to find a proper solution for Eq. (20), the following notation is introduced,

$$
\left.\dot{\boldsymbol{q}}^{\mathrm{d}}\right|_{\text {d.p. }}=-\left.\frac{\boldsymbol{m} \ddot{q}^{\mathrm{i}}}{\boldsymbol{k} \dot{\boldsymbol{q}}^{\mathrm{d}}}\right|_{\text {d.p. }}
$$

where

$$
\begin{aligned}
& \boldsymbol{m}=\left\{\begin{array}{llll}
m_{1} & m_{2} & \ldots & m_{n}
\end{array}\right\}^{\mathrm{T}}=\left.\operatorname{adj}\left(\boldsymbol{\phi}_{q}^{\mathrm{d}}\right) \boldsymbol{\phi}_{q}^{\mathrm{i}}\right|_{\text {d.p. }} \\
& \boldsymbol{k}=\left\{\begin{array}{llll}
k_{1} & k_{2} & \ldots & k_{n}
\end{array}\right\}=\left.\frac{\partial}{\partial \boldsymbol{q}^{\mathrm{d}}}\left(\operatorname{det}\left(\boldsymbol{\phi}_{q}^{\mathrm{d}}\right)\right)\right|_{\text {d.p. }}
\end{aligned}
$$


The generic solution for the $j$ th value of the $\dot{\boldsymbol{q}}^{\mathrm{d}}$ vector is

$$
\left.\dot{q}_{j}\right|_{\text {d.p. }}=-\left.\frac{m_{j}}{k_{1} \dot{q}_{1}+k_{2} \dot{q}_{2}+\cdots+k_{j} \dot{q}_{j}+\cdots+k_{n} \dot{q}_{n}} \ddot{q}^{\mathrm{i}}\right|_{\text {d.p. }}
$$

Thus, the quotient between two velocities $\dot{q}_{j}$ and $\dot{q}_{k}$ is

$$
\left.\frac{\dot{q}_{j}}{\dot{q}_{k}}\right|_{\text {d.p. }}=\left.\frac{m_{j}}{m_{k}}\right|_{\text {d.p. }}
$$

Combining both Eqs. (24) and (25) yields to the final generic solution

$$
\left.\dot{q}_{j}\right|_{\text {d.p. }}=\left.\sqrt{-\frac{m_{j}^{2}}{k m} \ddot{q}^{i}}\right|_{\text {d.p. }}
$$

which in vector form can be written as

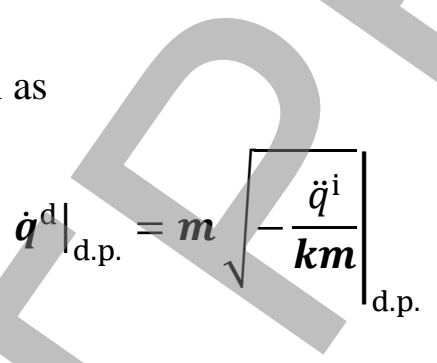

For the slider-crank mechanism considered in this study,

$$
\boldsymbol{\phi}\left(q_{1}, q_{2}, q_{3}\right)=\left\{\begin{array}{l}
\phi_{1}\left(q_{1}, q_{2}, q_{3}\right) \\
\phi_{2}\left(q_{1}, q_{2}, q_{3}\right)
\end{array}\right\}=\left\{\begin{array}{c}
r \sin q_{1}-l \sin q_{2} \\
-r \cos q_{1}-l \cos q_{2}+q_{3}
\end{array}\right\}=0
$$

with $\phi_{q}^{\mathrm{d}}$ and $\boldsymbol{\phi}_{q}^{\mathrm{i}}$ defined according to Eqs. (6) and (7)

$$
\begin{gathered}
\boldsymbol{\phi}_{q}^{\mathrm{d}}=\left[\begin{array}{cc}
r \cos q_{1} & -l \cos q_{2} \\
r \sin q_{1} & l \sin q_{2}
\end{array}\right] \\
\boldsymbol{\phi}_{q}^{\mathrm{i}}=\left\{\begin{array}{l}
0 \\
1
\end{array}\right\}
\end{gathered}
$$

Thus, at the dead point configuration defined in Figure $4 \mathrm{~b}$ )

$$
\left.\begin{array}{c}
\boldsymbol{m}=\left\{\begin{array}{l}
l \\
r
\end{array}\right\} \\
\boldsymbol{k}=\{r l \quad r l
\end{array}\right\}
$$

And, therefore, the velocities $\dot{q}_{1}$ and $\dot{q}_{2}$ are 


$$
\begin{aligned}
& \left.\dot{q}_{1}\right|_{\text {d.p. }}=\sqrt{-\frac{l}{r(r+l)} \ddot{q}_{3}} \\
& \left.\dot{q}_{2}\right|_{\text {d.p. }}=\sqrt{-\frac{r}{l(r+l)} \ddot{q}_{3}}
\end{aligned}
$$

In absence of bifurcations, the functions that define the constraint equations are always continuous and differentiable within the accessible configuration range of the mechanism. Therefore, $m_{j}^{2} /(\boldsymbol{k m})$ will be a finite and determined value. According to Eq. (27), the velocities of the dependent coordinates are proportional to the square root of the acceleration of the independent coordinate at the configuration of dead point. Consequently, assuming that a conventional electrical actuator is able to perform a motion with a minimum $\mathcal{C}^{1}$ continuity, the acceleration $\ddot{q}^{\mathrm{i}}$ of the independent coordinate must be, at least, continuous around this point $\left(\mathcal{C}^{0}\right)$. Therefore, the motion law described by the time evolution of the independent coordinate $q^{\mathrm{i}}$ must be, at least, $\mathcal{C}^{2}$. Otherwise, with a lower continuity degree, the velocities of the dependent coordinates $\dot{\boldsymbol{q}}^{\mathrm{d}}$ will change instantaneously between two different values, making the movement not feasible for conventional actuators.

\subsection{Acceleration analysis}

The acceleration analysis is based on Eq. (14). When the independent coordinate is located near a dead point, the numerator of this expression tends to zero (see Appendix A). Therefore, there is an algebraic indeterminacy in the calculation of $\ddot{\boldsymbol{q}}^{\mathrm{d}}$, which is solved again using L'Hôpital's rule.

$$
\begin{gathered}
\left.\ddot{\boldsymbol{q}}^{\mathrm{d}}\right|_{\text {d.p. }}=\lim _{q^{\mathrm{i}} \rightarrow \text { d.p. }}\left(-\frac{\frac{\mathrm{d}}{\mathrm{d} t}\left(\operatorname{adj}\left(\boldsymbol{\phi}_{q}^{\mathrm{d}}\right)\left[\boldsymbol{\phi}_{q}^{\mathrm{i}} \ddot{q}^{\mathrm{i}}+\dot{\boldsymbol{\phi}}_{q} \dot{\boldsymbol{q}}\right]\right)}{\frac{\mathrm{d}}{\mathrm{d} t}\left(\operatorname{det}\left(\boldsymbol{\phi}_{q}^{\mathrm{d}}\right)\right)}\right)= \\
=\lim _{q^{\mathrm{i} \rightarrow \text { d.p. }}}\left(\frac{\frac{\mathrm{d}}{\mathrm{d} t}\left(\operatorname{adj}\left(\boldsymbol{\phi}_{q}^{\mathrm{d}}\right)\right)\left[\boldsymbol{\phi}_{q}^{\mathrm{i}} \ddot{q}^{\mathrm{i}}+\dot{\boldsymbol{\phi}}_{q} \dot{\boldsymbol{q}}\right]+\operatorname{adj}\left(\boldsymbol{\phi}_{q}^{\mathrm{d}}\right)\left[\dot{\boldsymbol{\phi}}_{q}^{\mathrm{i}} \ddot{q}^{\mathrm{i}}+\boldsymbol{\phi}_{q}^{\mathrm{i}} \ddot{q}^{\mathrm{i}}+\ddot{\boldsymbol{\phi}}_{q} \dot{\boldsymbol{q}}+\dot{\boldsymbol{\phi}}_{q} \ddot{\boldsymbol{q}}\right]}{\left\{\frac{\partial}{\partial \boldsymbol{q}^{\mathrm{d}}}\left(\operatorname{det}\left(\boldsymbol{\phi}_{q}^{\mathrm{d}}\right)\right), \frac{\partial}{\partial q^{\mathrm{i}}}\left(\operatorname{det}\left(\boldsymbol{\phi}_{q}^{\mathrm{d}}\right)\right)\right\}\left\{\dot{\boldsymbol{q}}^{\mathrm{d}}, \dot{q}^{\mathrm{i}}\right\}^{\mathrm{T}}}\right)
\end{gathered}
$$

Splitting the dependent and independent terms and imposing $\dot{q}^{\mathrm{i}}=0$

$$
\left.\ddot{\boldsymbol{q}}^{\mathrm{d}}\right|_{\text {d.p. }}=-\left.\frac{\frac{\mathrm{d}}{\mathrm{d} t}\left(\operatorname{adj}\left(\boldsymbol{\phi}_{q}^{\mathrm{d}}\right)\right)\left[\boldsymbol{\phi}_{q}^{\mathrm{i}} \ddot{q}^{\mathrm{i}}+\dot{\boldsymbol{\phi}}_{q}^{\mathrm{d}} \dot{\boldsymbol{q}}^{\mathrm{d}}\right]+\operatorname{adj}\left(\boldsymbol{\phi}_{q}^{\mathrm{d}}\right)\left[2 \dot{\boldsymbol{\phi}}_{q}^{\mathrm{i}} \ddot{q}^{\mathrm{i}}+\boldsymbol{\phi}_{q}^{\mathrm{i}} \ddot{q}^{\mathrm{i}}+\ddot{\boldsymbol{\phi}}_{q}^{\mathrm{d}} \dot{\boldsymbol{q}}^{\mathrm{d}}+\dot{\boldsymbol{\phi}}_{q}^{\mathrm{d}} \ddot{\boldsymbol{q}}^{\mathrm{d}}\right]}{\frac{\partial}{\partial \boldsymbol{q}^{\mathrm{d}}}\left(\operatorname{det}\left(\boldsymbol{\phi}_{q}^{\mathrm{d}}\right)\right) \dot{\boldsymbol{q}}^{\mathrm{d}}}\right|_{\text {d.p. }}
$$

where (see Appendix B for expressions (38) and (39)) 


$$
\begin{gathered}
\left.\dot{\boldsymbol{\phi}}_{q}^{\mathrm{i}}\right|_{\text {d.p. }}=\left.\frac{\partial}{\partial \boldsymbol{q}^{\mathrm{d}}}\left(\boldsymbol{\phi}_{q}^{\mathrm{i}}\right) \dot{\boldsymbol{q}}^{\mathrm{d}}\right|_{\text {d.p. }}=\left.\left(\boldsymbol{\phi}_{q}^{\mathrm{i}}\right)^{\mathrm{d}} \dot{\boldsymbol{q}}^{\mathrm{d}}\right|_{\text {d.p. }} \\
\left.\dot{\boldsymbol{\phi}}_{q}^{\mathrm{d}}\right|_{\text {d.p. }}=\left.\sum_{k=1}^{n}\left(\frac{\partial}{\partial q_{k}}\left(\boldsymbol{\phi}_{q}^{\mathrm{d}}\right) \dot{q}_{k}\right)\right|_{\text {d.p. }} \\
\left.\ddot{\boldsymbol{\phi}}_{q}^{\mathrm{d}}\right|_{\text {d.p. }}=\sum_{l=1}^{n}\left(\sum_{k=1}^{n}\left(\frac{\partial}{\partial q_{k}}\left(\frac{\partial}{\partial q_{l}}\left(\boldsymbol{\phi}_{q}^{\mathrm{d}}\right)\right) \dot{q}_{k}\right) \dot{q}_{l}+\frac{\partial}{\partial q_{l}}\left(\boldsymbol{\phi}_{q}^{\mathrm{d}}\right) \ddot{q}_{l}\right)+ \\
+\left.\frac{\partial}{\partial q^{\mathrm{i}}}\left(\boldsymbol{\phi}_{q}^{\mathrm{d}}\right) \ddot{q}^{\mathrm{i}}\right|_{\text {d.p. }}
\end{gathered}
$$

In order to facilitate the notation, $\left.\ddot{\boldsymbol{\phi}}_{q}^{\mathrm{d}}\right|_{\text {d.p. }}$ is split into three matrices

$$
\begin{gathered}
\left.\left(\ddot{\boldsymbol{\phi}}_{q}^{\mathrm{d}}\right)_{1}\right|_{\text {d.p. }}=\left.\sum_{l=1}^{n}\left(\sum_{k=1}^{n}\left(\frac{\partial}{\partial q_{k}}\left(\frac{\partial}{\partial q_{l}}\left(\boldsymbol{\phi}_{q}^{\mathrm{d}}\right)\right) \dot{q}_{k}\right) \dot{q}_{l}\right)\right|_{\text {d.p. }} \\
\left.\left(\ddot{\boldsymbol{\phi}}_{q}^{\mathrm{d}}\right)_{2}\right|_{\text {d.p. }}=\left.\frac{\partial}{\partial q^{\mathrm{i}}}\left(\boldsymbol{\phi}_{q}^{\mathrm{d}}\right) \ddot{q}^{\mathrm{i}}\right|_{\text {d.p. }} \\
\left.\left(\ddot{\boldsymbol{\phi}}_{q}^{\mathrm{d}}\right)_{3}\right|_{\text {d.p. }}=\left.\sum_{k=1}^{n}\left(\frac{\partial}{\partial q_{k}}\left(\boldsymbol{\phi}_{q}^{\mathrm{d}}\right) \ddot{q}_{k}\right)\right|_{\text {d.p. }}
\end{gathered}
$$

Rearranging terms in Eq. (36)

$$
\begin{gathered}
\frac{\partial}{\partial \boldsymbol{q}^{\mathrm{d}}}\left(\operatorname{det}\left(\boldsymbol{\phi}_{q}^{\mathrm{d}}\right)\right) \dot{\boldsymbol{q}}^{\mathrm{d}} \ddot{\boldsymbol{q}}^{\mathrm{d}}+\operatorname{adj}\left(\boldsymbol{\phi}_{q}^{\mathrm{d}}\right)\left(\ddot{\boldsymbol{\phi}}_{q}^{\mathrm{d}}\right)_{3} \dot{\boldsymbol{q}}^{\mathrm{d}}+\left.\operatorname{adj}\left(\boldsymbol{\phi}_{q}^{\mathrm{d}}\right) \dot{\boldsymbol{\phi}}_{q}^{\mathrm{d}} \ddot{\boldsymbol{q}}^{\mathrm{d}}\right|_{\mathrm{d} . \mathrm{p} .}= \\
=-\left(\frac{\mathrm{d}}{\mathrm{d} t}\left(\operatorname{adj}\left(\boldsymbol{\phi}_{q}^{\mathrm{d}}\right)\right)\left[\boldsymbol{\phi}_{q}^{\mathrm{i}} \ddot{q}^{\mathrm{i}}+\dot{\boldsymbol{\phi}}_{q}^{\mathrm{d}} \dot{\boldsymbol{q}}^{\mathrm{d}}\right]+\right. \\
\left.+\operatorname{adj}\left(\boldsymbol{\phi}_{q}^{\mathrm{d}}\right)\left[2 \frac{\partial}{\partial \boldsymbol{q}^{\mathrm{d}}}\left(\boldsymbol{\phi}_{q}^{\mathrm{i}}\right) \dot{\boldsymbol{q}}^{\mathrm{d}} \ddot{q}^{\mathrm{i}}+\boldsymbol{\phi}_{q}^{\mathrm{i}} \ddot{q}^{\mathrm{i}}+\left(\left(\ddot{\boldsymbol{\phi}}_{q}^{\mathrm{d}}\right)_{1}+\left(\ddot{\boldsymbol{\phi}}_{q}^{\mathrm{d}}\right)_{2}\right) \dot{\boldsymbol{q}}^{\mathrm{d}}\right]\right)\left.\right|_{\text {d.p. }}
\end{gathered}
$$

In order to see the dependence between the acceleration of the dependent coordinates $\ddot{\boldsymbol{q}}^{\mathrm{d}}$ and the motion of the independent coordinate $q^{\mathrm{i}}$, Eqs. (37), (38) and (40) are rewritten considering Eqs. $(25-27)$

$$
\begin{gathered}
\left.\dot{\boldsymbol{\phi}}_{q}^{\mathrm{i}}\right|_{\text {d.p. }}=\left.\left(\boldsymbol{\phi}_{q}^{\mathrm{i}}\right)^{d} \boldsymbol{m} \sqrt{\frac{-\ddot{q}^{\mathrm{i}}}{\boldsymbol{k m}}}\right|_{\text {d.p. }} \\
\left.\dot{\boldsymbol{\phi}}_{q}^{\mathrm{d}}\right|_{\text {d.p. }}=\sum_{k=1}^{n}\left(\frac{\partial}{\partial q_{k}}\left(\boldsymbol{\phi}_{q}^{\mathrm{d}}\right) m_{k}\right) \sqrt{\left.\frac{-\ddot{q}^{\mathrm{i}}}{\boldsymbol{k \boldsymbol { m }}}\right|_{\text {d.p. }}}
\end{gathered}
$$




$$
\left.\left(\ddot{\boldsymbol{\phi}}_{q}^{\mathrm{d}}\right)_{1}\right|_{\text {d.p. }}=\left.\sum_{l=1}^{n}\left(\sum_{k=1}^{n}\left(\frac{\partial}{\partial q_{k}}\left(\frac{\partial}{\partial q_{l}}\left(\boldsymbol{\phi}_{q}^{\mathrm{d}}\right)\right) m_{k}\right) m_{l}\right) \frac{-\ddot{q}^{\mathrm{i}}}{\boldsymbol{k} \boldsymbol{m}}\right|_{\text {d.p. }}
$$

Therefore, Eq. (43) is rewritten, considering Eq. (23), (27), (41), (42) and (44)-(46). For the calculation of the derivative of the adjoint matrix of $\boldsymbol{\phi}_{q}^{\mathrm{d}}$, Eq. (45) is used, replacing $\boldsymbol{\phi}_{q}^{\mathrm{d}}$ with $\operatorname{adj}\left(\boldsymbol{\phi}_{q}^{\mathrm{d}}\right)$.

$$
\begin{gathered}
\boldsymbol{k} \boldsymbol{m} \ddot{\boldsymbol{q}}^{\mathrm{d}}+\left.\operatorname{adj}\left(\boldsymbol{\phi}_{q}^{\mathrm{d}}\right)\left[\sum_{k=1}^{n}\left(\frac{\partial}{\partial q_{k}}\left(\boldsymbol{\phi}_{q}^{\mathrm{d}}\right) \ddot{q}_{k}\right) \boldsymbol{m}+\sum_{k=1}^{n}\left(\frac{\partial}{\partial q_{k}}\left(\boldsymbol{\phi}_{q}^{\mathrm{d}}\right) m_{k}\right) \ddot{\boldsymbol{q}}^{\mathrm{d}}\right]\right|_{\mathrm{d} . \mathbf{p} .}= \\
=-\left[\boldsymbol{m} \sqrt{\left.\frac{\boldsymbol{k} \boldsymbol{m}}{-\ddot{q}^{\mathrm{i}}} \dddot{q}^{\mathrm{i}}+\boldsymbol{R} \ddot{q}^{\mathrm{i}}\right]\left.\right|_{\text {d.p. }}}\right.
\end{gathered}
$$

with the vector $\left.\boldsymbol{R}\right|_{\text {d.p. }}$ being

$$
\begin{gathered}
\left.\boldsymbol{R}\right|_{\text {d.p. }}=\sum_{k=1}^{n}\left(\frac{\partial}{\partial q_{k}}\left(\operatorname{adj}\left(\boldsymbol{\phi}_{q}^{\mathrm{d}}\right)\right) m_{k}\right)\left[\boldsymbol{\phi}_{q}^{\mathrm{i}}-\frac{1}{\boldsymbol{k} \boldsymbol{m}} \sum_{k=1}^{n}\left(\frac{\partial}{\partial q_{k}}\left(\boldsymbol{\phi}_{q}^{\mathrm{d}}\right) m_{k}\right) \boldsymbol{m}\right]+ \\
+\left.\operatorname{adj}\left(\boldsymbol{\phi}_{q}^{\mathrm{d}}\right)\left[2\left(\boldsymbol{\phi}_{q}^{\mathrm{i}}\right)^{d}-\frac{1}{\boldsymbol{k} \boldsymbol{m}} \sum_{l=1}^{n}\left(\sum_{k=1}^{n}\left(\frac{\partial}{\partial q_{k}}\left(\frac{\partial}{\partial q_{l}}\left(\boldsymbol{\phi}_{q}^{\mathrm{d}}\right)\right) m_{k}\right) m_{l}\right)+\frac{\partial}{\partial q^{\mathrm{i}}}\left(\boldsymbol{\phi}_{q}^{\mathrm{d}}\right)\right] \boldsymbol{m}\right|_{\text {d.p. }}
\end{gathered}
$$

Regrouping terms,

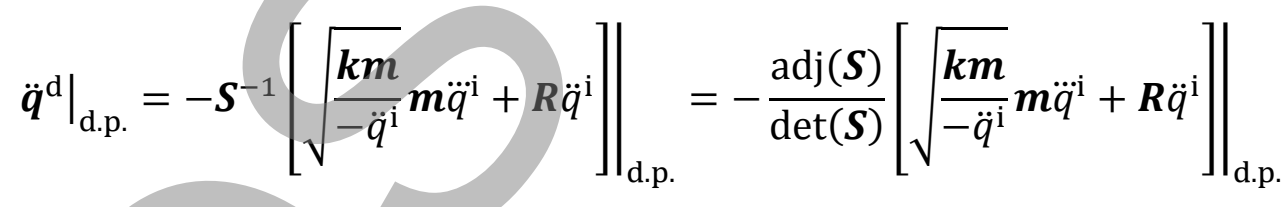

with the matrix $\left.\boldsymbol{S}\right|_{\text {d.p. }}$ defined as follows

$$
\begin{gathered}
\left.\boldsymbol{S}\right|_{\text {d.p. }}=\boldsymbol{k} \boldsymbol{m} \boldsymbol{I}+ \\
+\operatorname{adj}\left(\boldsymbol{\phi}_{q}^{\mathrm{d}}\right)\left[\left[\begin{array}{llll}
\frac{\partial}{\partial q_{1}}\left(\boldsymbol{\phi}_{q}^{\mathrm{d}}\right) \boldsymbol{m} & \frac{\partial}{\partial q_{2}}\left(\boldsymbol{\phi}_{q}^{\mathrm{d}}\right) \boldsymbol{m} & \cdots & \frac{\partial}{\partial q_{n}}\left(\boldsymbol{\phi}_{q}^{\mathrm{d}}\right) \boldsymbol{m}
\end{array}\right]+\sum_{k=1}^{n}\left(\frac{\partial}{\partial q_{k}}\left(\boldsymbol{\phi}_{q}^{\mathrm{d}}\right) m_{k}\right)\right]
\end{gathered}
$$

where $I$ is an $n$ by $n$ identity matrix. Based on the previous subsection 4.1 , the law of motion of the independent coordinate must be, at least, $\mathcal{C}^{2}$. This implies that the acceleration $\ddot{q}^{i}$ must be continuous around the dead point. According to Eq. (49), the calculation of the dependent 
accelerations $\ddot{\boldsymbol{q}}^{\mathrm{d}}$ may considerably differ if the acceleration $\ddot{q}^{\mathrm{i}}$ of the independent coordinate is zero or has any finite value, because it appears in the denominator of the equation. These two scenarios are analyzed in the following subsections.

\subsubsection{Acceleration $\ddot{q}^{\mathrm{i}} \neq 0$}

When the mechanism reaches a dead point configuration with an acceleration of the independent coordinate $\ddot{q}^{\mathrm{i}}$ different than zero, and assuming $\operatorname{det}(\boldsymbol{S}) \neq 0$, the acceleration of the dependent coordinates $\ddot{\boldsymbol{q}}^{\mathrm{d}}$ could directly be obtained from Eq. (49). As conventional electrical actuators can perform $\mathcal{C}^{1}$ input commands, there are no requirements with respect to the jerk $\dddot{q}^{\mathrm{i}}$, as long as it has a finite value. Therefore, if $\ddot{q}^{\mathrm{i}} \neq 0$, the motion law of the independent coordinate must be, at least, $\mathcal{C}^{2}$.

In the case of the slider-crank mechanism considered throughout this study

Thus,

$$
\begin{gathered}
\left.\boldsymbol{S}\right|_{\text {d.p. }}=\left[\begin{array}{cc}
r^{2} l+3 r l^{2} & 2 r l^{2} \\
2 r^{2} l & 3 r^{2} l+r l^{2}
\end{array}\right] \\
\left.\boldsymbol{R}\right|_{\text {d.p. }}=\left\{\begin{array}{l}
0 \\
0
\end{array}\right\}
\end{gathered}
$$

\subsubsection{Acceleration $\ddot{q}^{\mathrm{i}}=0$}

$$
\begin{aligned}
& \left.\ddot{q}_{1}\right|_{\text {d.p. }}=-\frac{l \dddot{q}_{3}}{3 \sqrt{-\ddot{q}_{3} r l(r+l)}} \\
& \left.\ddot{q}_{2}\right|_{\text {d.p. }}=-\frac{r \ddot{q}_{3}}{3 \sqrt{-\ddot{q}_{3} r l(r+l)}}
\end{aligned}
$$

Based on Eq. (49), when the mechanism is located at a dead point with a null $\ddot{q}^{\mathrm{i}}$ acceleration, the value of the jerk $\dddot{q}^{\mathrm{i}}$ must also be null. Otherwise, the accelerations $\ddot{\boldsymbol{q}}^{\mathrm{d}}$ of the dependent coordinates will tend to infinity. Moreover, as $\ddot{q}^{\mathrm{i}}=0$, the velocities $\dot{\boldsymbol{q}}^{\mathrm{d}}$ are also null. Therefore, to know the exact value of $\ddot{\boldsymbol{q}}^{\mathrm{d}}$ when $\dot{\boldsymbol{q}}^{\mathrm{d}}=\dot{q}^{\mathrm{i}}=\ddot{q}^{\mathrm{i}}=\dddot{q}^{\mathrm{i}}=0$, L'Hôpital's rule is again applied to Eq. (35)

$\left.\ddot{q}^{\mathrm{d}}\right|_{\text {d.p. }}=\lim _{q^{\mathrm{i}} \rightarrow \text { d.p. }}\left(-\frac{\frac{\mathrm{d}}{\mathrm{d} t}\left(\frac{\mathrm{d}}{\mathrm{d} t}\left(\operatorname{adj}\left(\boldsymbol{\phi}_{q}^{\mathrm{d}}\right)\right)\left[\boldsymbol{\phi}_{q}^{\mathrm{i}} \ddot{q}^{\mathrm{i}}+\dot{\boldsymbol{\phi}}_{q} \dot{\boldsymbol{q}}\right]+\operatorname{adj}\left(\boldsymbol{\phi}_{q}^{\mathrm{d}}\right)\left[\dot{\boldsymbol{\phi}}_{q}^{\mathrm{i}} \ddot{q}^{\mathrm{i}}+\boldsymbol{\phi}_{q}^{\mathrm{i}} \ddot{\boldsymbol{q}}^{\mathrm{i}}+\ddot{\boldsymbol{\phi}}_{q} \dot{\boldsymbol{q}}+\dot{\boldsymbol{\phi}}_{q} \ddot{\boldsymbol{q}}\right]\right)}{\frac{\mathrm{d}}{\mathrm{d} t}\left(\left\{\frac{\partial}{\partial \boldsymbol{q}^{\mathrm{d}}}\left(\operatorname{det}\left(\boldsymbol{\phi}_{q}^{\mathrm{d}}\right)\right), \frac{\partial}{\partial q^{\mathrm{i}}}\left(\operatorname{det}\left(\boldsymbol{\phi}_{q}^{\mathrm{d}}\right)\right)\right\}\left\{\dot{\boldsymbol{q}}^{\mathrm{d}}, \dot{q}^{\mathrm{i}}\right\}^{\mathrm{T}}\right)}\right)$

Splitting again the dependent and independent terms and imposing $\dot{\boldsymbol{q}}^{\mathrm{d}}=\dot{q}^{\mathrm{i}}=\ddot{q}^{\mathrm{i}}=\dddot{q}^{\mathrm{i}}=0$ 


$$
\left.\ddot{\boldsymbol{q}}^{\mathrm{d}}\right|_{\text {d.p. }}=-\left.\frac{\operatorname{adj}\left(\boldsymbol{\phi}_{q}^{\mathrm{d}}\right)\left[\boldsymbol{\phi}_{q}^{\mathrm{i}} \dddot{q}^{\mathrm{i}}+2 \ddot{\boldsymbol{\phi}}_{q}^{\mathrm{d}} \ddot{\boldsymbol{q}}^{\mathrm{d}}\right]}{\frac{\mathrm{d}}{\mathrm{d} t}\left(\frac{\partial}{\partial \boldsymbol{q}^{\mathrm{d}}}\left(\operatorname{det}\left(\boldsymbol{\phi}_{q}^{\mathrm{d}}\right)\right) \dot{\boldsymbol{q}}^{\mathrm{d}}\right)}\right|_{\text {d.p. }}=-\left.\frac{\operatorname{adj}\left(\boldsymbol{\phi}_{q}^{\mathrm{d}}\right)\left[\boldsymbol{\phi}_{q}^{\mathrm{i}} \dddot{q}^{\mathrm{i}}+2 \ddot{\boldsymbol{\phi}}_{q}^{\mathrm{d}} \ddot{\boldsymbol{q}}^{\mathrm{d}}\right]}{\frac{\mathrm{d}}{\mathrm{d} t}\left(\boldsymbol{k} \dot{\boldsymbol{q}}^{\mathrm{d}}\right)}\right|_{\text {d.p. }}
$$

Considering Eq. (39) and $\dot{\boldsymbol{q}}^{\mathrm{d}}=0$

$$
\left(\boldsymbol{k} \ddot{\boldsymbol{q}}^{\mathrm{d}}\right) \ddot{\boldsymbol{q}}^{\mathrm{d}}+\left.2 \operatorname{adj}\left(\boldsymbol{\phi}_{q}^{\mathrm{d}}\right) \sum_{l=1}^{n}\left(\frac{\partial}{\partial q_{l}}\left(\boldsymbol{\phi}_{q}^{\mathrm{d}}\right) \ddot{q}_{l}\right) \ddot{\boldsymbol{q}}^{\mathrm{d}}\right|_{\text {d.p. }}=-\left.\boldsymbol{m} \dddot{q}^{\mathrm{i}}\right|_{\mathrm{d} . \mathrm{p} .}
$$

The relation between the $j$ th and the $k$ th value of the acceleration vector $\ddot{\boldsymbol{q}}^{\mathrm{d}}$ is obtained by differentiating Eq. (5) and applying twice L'Hôpital's rule considering $\dot{q}^{\mathrm{i}}=\ddot{q}^{\mathrm{i}}=\dddot{q}^{\mathrm{i}}=0$

$$
\left.\frac{\ddot{q}_{j}}{\ddot{q}_{k}}\right|_{\text {d.p. }}=\left.\frac{m_{j}}{m_{k}}\right|_{\text {d.p. }}
$$

Thus, for the $j$ th component of the acceleration $\ddot{\boldsymbol{q}}^{d}$

$$
\boldsymbol{k} \boldsymbol{m} \frac{\ddot{q}_{j}}{m_{j}} \ddot{\boldsymbol{q}}^{d}+\left.2 \operatorname{adj}\left(\boldsymbol{\phi}_{q}^{\mathrm{d}}\right) \sum_{k=1}^{n}\left(\frac{\partial}{\partial q_{k}}\left(\boldsymbol{\phi}_{q}^{\mathrm{d}}\right) m_{k}\right) \boldsymbol{m}\left(\frac{\ddot{q}_{j}}{m_{j}}\right)^{2}\right|_{\mathrm{d} . \mathrm{p} .}=-\left.\boldsymbol{m} \dddot{q}^{\mathrm{i}}\right|_{\mathrm{d} . \mathrm{p} .}
$$

Therefore, considering the $j$ th row of Eq. (59), its final expression is

$$
\left.\ddot{q}_{j}\right|_{\text {d.p. }}=\left.\sqrt{\frac{-m_{j}^{3} \dddot{q}^{\mathrm{i}}}{m_{j} \boldsymbol{k} \boldsymbol{m}+2 \boldsymbol{a}_{\boldsymbol{j} *} \sum_{k=1}^{n}\left(\frac{\partial}{\partial q_{k}}\left(\boldsymbol{\phi}_{q}^{\mathrm{d}}\right) m_{k}\right) \boldsymbol{m}}}\right|_{\text {d.p. }}
$$

where $\boldsymbol{a}_{\boldsymbol{j} *}$ is the $j$ th row of the matrix adj $\left(\boldsymbol{\phi}_{q}^{\mathrm{d}}\right)$.

In the case of the slider-crank mechanism, in the configuration of dead point shown in Figure 4 b)

$$
\begin{aligned}
& \left.\ddot{q}_{1}\right|_{\text {d.p. }}=\sqrt{\frac{-l}{3 r(r+l)} \dddot{q}_{3}} \\
& \left.\ddot{q}_{2}\right|_{\text {d.p. }}=\sqrt{\frac{-r}{3 l(r+l)} \dddot{q}_{3}}
\end{aligned}
$$


According to Eq. (60), the acceleration of each of the $n$ dependent coordinates is proportional to the square root of $\dddot{q}^{\mathrm{i}}$. Based on section 3 , conventional actuators can perform motions with a discontinuity in the acceleration. Therefore, $\dddot{q}^{\mathrm{i}}$ can be any finite value, but it is not necessary to be continuous. Thus, if $\ddot{q}^{\mathrm{i}}=0$, the motion law of the independent coordinate must be, at least, $\mathcal{C}^{3}$, with $\dddot{q}^{\mathrm{i}}$ necessarily zero.

\section{Simulation}

This section presents the simulations carried out to verify the results obtained in section 4 . For this purpose, the two configurations presented in Figure 2b, a four linkage mechanism, and Figure $4 \mathrm{~b}$, a slider-crank mechanism, are studied. For the first case, the lengths of the mechanism are $l_{1}=0.075 \mathrm{~m}, l_{2}=0.175 \mathrm{~m}, l_{3}=0.1 \mathrm{~m}$ and $l_{4}=0.175 \mathrm{~m}$. Regarding the slider-crank mechanism, the values for $r$ and $l$ are $0.75 \mathrm{~m}$ and $1.25 \mathrm{~m}$, respectively. The instant of time in which the mechanism is found at the dead point configuration is set to $t_{0}=0$.

Two situations have been analyzed. The first case, based on 4.2.2, shows the influence of the value $\dddot{q}^{\mathrm{i}}$ of the independent coordinate when its acceleration $\ddot{q}^{\mathrm{i}}$ is null at the dead point configuration. The second part of the simulation, referring to 4.2.1., considers a motion law with $\ddot{q}^{\mathrm{i}} \neq 0$ near the dead point. The motion law of the independent coordinate $q_{3}$ around the dead point follows Eq. (63)

$$
q_{3}(t)= \begin{cases}-c_{4} t^{4}+c_{3} t^{3}-c_{2} t^{2}+c_{1} t+c_{0} & -\infty<t<t_{0} \\ -c_{4} t^{4}-c_{3} t^{3}-c_{2} t^{2}+c_{1} t+c_{0} & t_{0} \leq t<+\infty\end{cases}
$$

where the values of $c_{0}, c_{1}, c_{2}, c_{3}$ and $c_{4}$, listed in Table 1 , depend on the case simulated and the mechanism used.

\begin{tabular}{|c|c|c|c|c|c|c|}
\hline & \multicolumn{3}{|c|}{ Four linkage } & \multicolumn{3}{c|}{ Slider-crank } \\
\cline { 2 - 7 } & $\ddot{q}_{3}\left(t_{0}\right)=0$ & $\ddot{q}_{3}\left(t_{0}\right)=0$ & $\ddot{q}_{3}\left(t_{0}\right) \neq 0$ & $\ddot{q}_{3}\left(t_{0}\right)=0$ & $\ddot{q}_{3}\left(t_{0}\right)=0$ & $\ddot{q}_{3}\left(t_{0}\right) \neq 0$ \\
& $\dddot{q}_{3}\left(t_{0}\right) \neq 0$ & $\dddot{q}_{3}\left(t_{0}\right)=0$ & $\dddot{q}_{3}\left(t_{0}\right) \neq 0$ & $\dddot{q}_{3}\left(t_{0}\right) \neq 0$ & $\dddot{q}_{3}\left(t_{0}\right)=0$ & $\dddot{q}_{3}\left(t_{0}\right) \neq 0$ \\
\hline$c_{0}$ & 2.25 & 2.25 & 2.25 & 2 & 2 & 2 \\
\hline$c_{1}$ & 0 & 0 & 0 & 0 & 0 & 0 \\
\hline$c_{2}$ & 0 & 0 & 0.5 & 0 & 0 & 0.5 \\
\hline$c_{3}$ & 0.563 & 0 & 0.563 & 0.5 & 0 & 0.5 \\
\hline$c_{4}$ & 0 & 0.563 & 0 & 0 & 0.5 & 0 \\
\hline
\end{tabular}

Table 1. Values of $c_{0}, c_{1}, c_{2}, c_{3}$ and $c_{4}$ for all the simulations. Units in SI. 


\subsection{Acceleration $\ddot{q}^{\mathrm{i}}=0$}

When the mechanism reaches a dead point configuration of the independent coordinate $q^{\mathrm{i}}$ with null acceleration $\ddot{q}^{\mathrm{i}}$, the jerk $\dddot{q}^{\mathrm{i}}$ must also be null. Otherwise, according to Eq. (49), the acceleration $\ddot{\boldsymbol{q}}^{\mathrm{d}}$ of the dependent coordinates will tend to infinity and the motion will not be feasible for conventional electrical actuators. This statement can be depicted in Figures 5 and 6.

As it can be seen in Figure 5, the acceleration $\ddot{q}_{3}$ is zero by the time the mechanism gets to the dead point configuration. Nevertheless, the absolute value of the jerk $\dddot{q}_{3}$ in this configuration is different than zero in both mechanisms. Thus, the accelerations $\ddot{q}_{1}$ and $\ddot{q}_{2}$ tend to infinity.
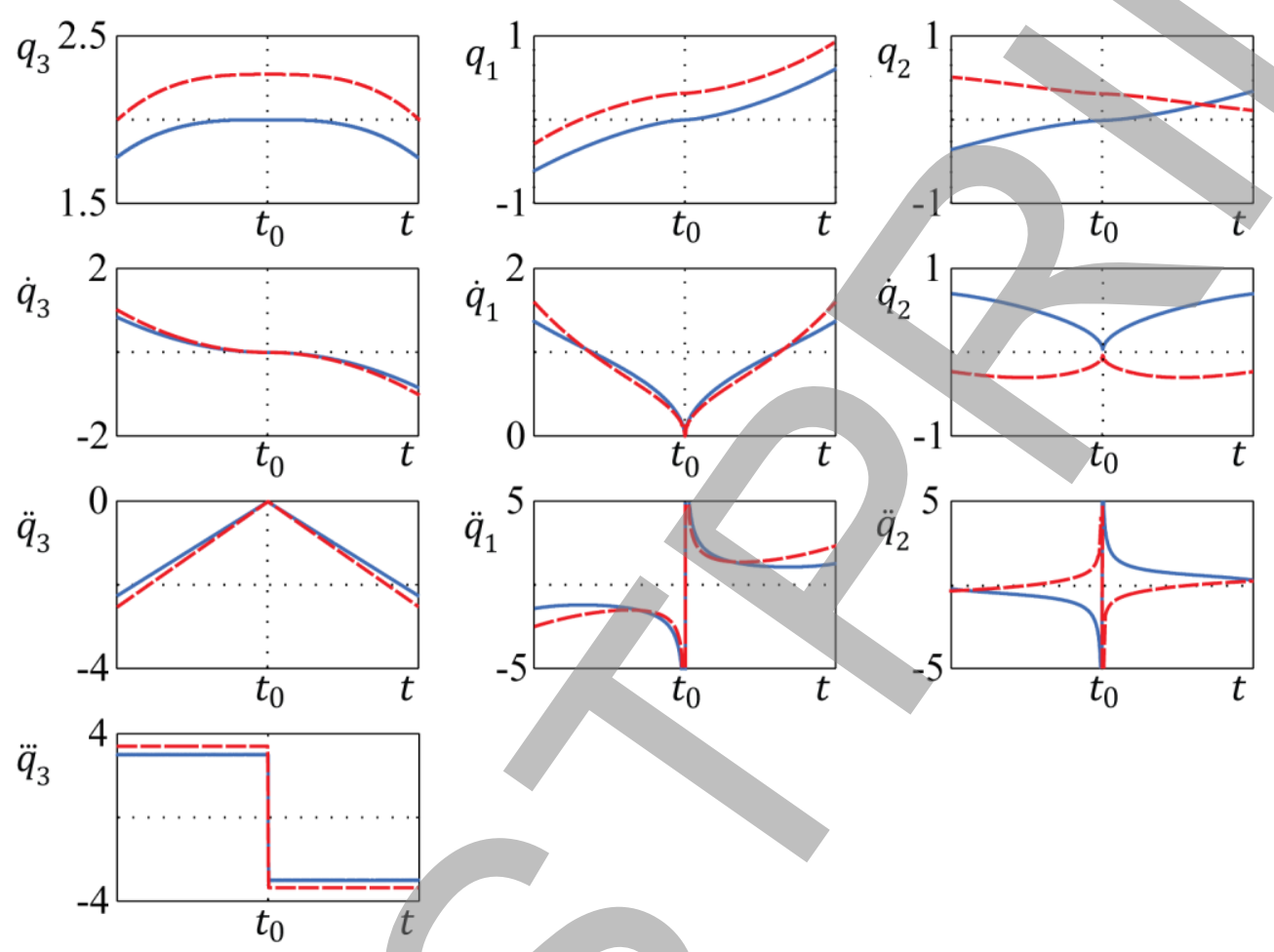

Figure 5: Motion law of $q_{3}$ with $\ddot{q}_{3}\left(t_{0}\right)=0$ and $\ddot{q}_{3}\left(t_{0}\right) \neq 0$ for the four linkage (dashed line) and slider-crank (solid line) mechanisms. Units in SI.

However, if the motion law of $q_{3}$ is modified so that the jerk $\dddot{q}_{3}$ is also null at $t_{0}$ (Figure 6), accelerations $\ddot{q}_{1}$ and $\ddot{q}_{2}$ are finite and can be calculated according to Eq. (60). 

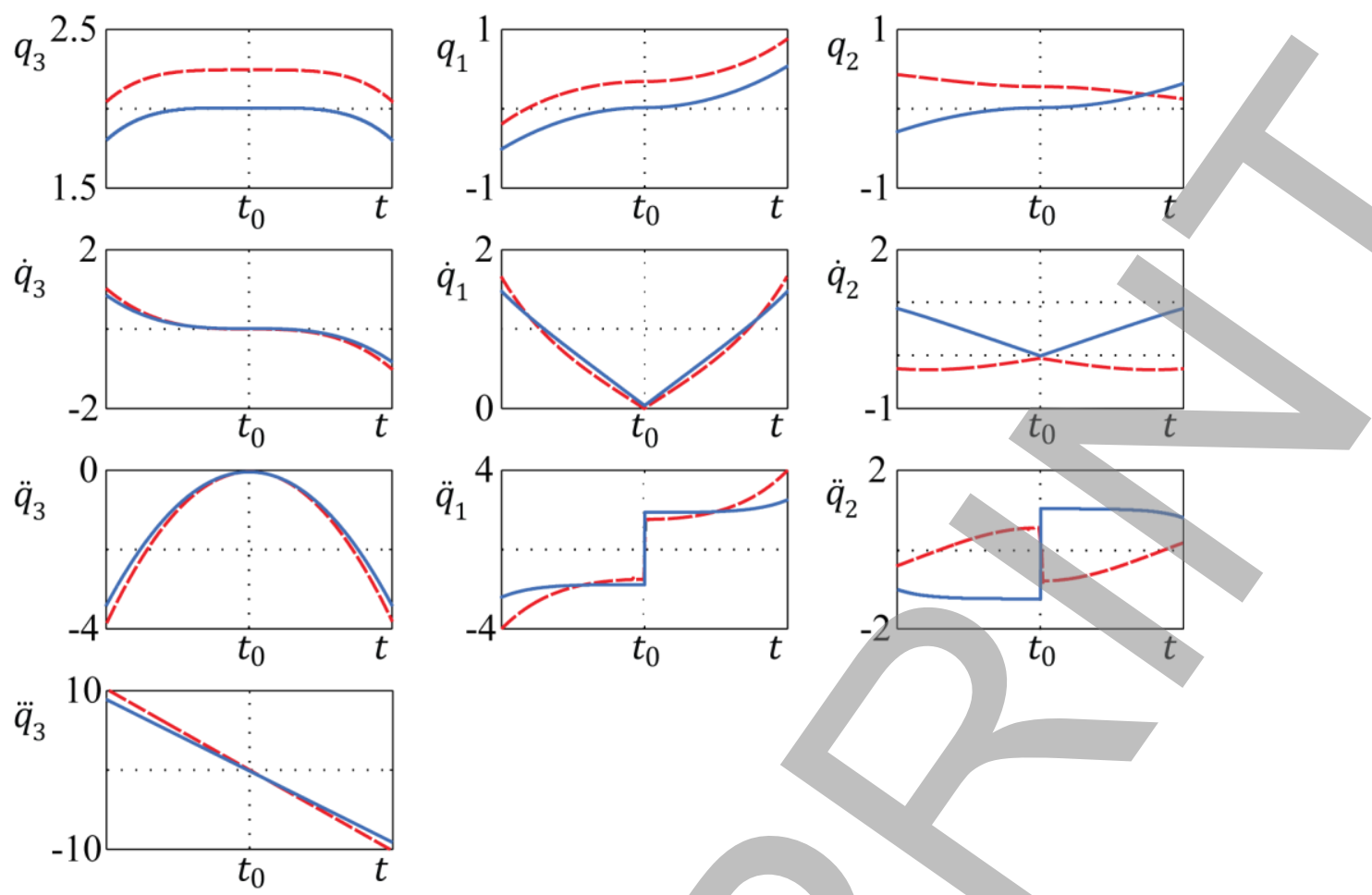

Figure 6: Motion law of $q_{3}$ with $\ddot{q}_{3}\left(t_{0}\right)=0$ and $\dddot{q}_{3}\left(t_{0}\right)=0$ for the four linkage (dashed line) and slider-crank (solid line) mechanisms. Units in SI.

\subsection{Acceleration $\ddot{q}^{\mathrm{i}} \neq 0$}

Unlike the two cases presented in 5.1, the acceleration $\ddot{q}_{3}$ has a finite value different than zero when the mechanism reaches the dead point configuration (Figure 7). Thus, accelerations $\ddot{q}_{1}$ and $\ddot{q}_{2}$ can be calculated using Eq. (49), regardless of the value of the jerk $\dddot{q}_{3}$. 

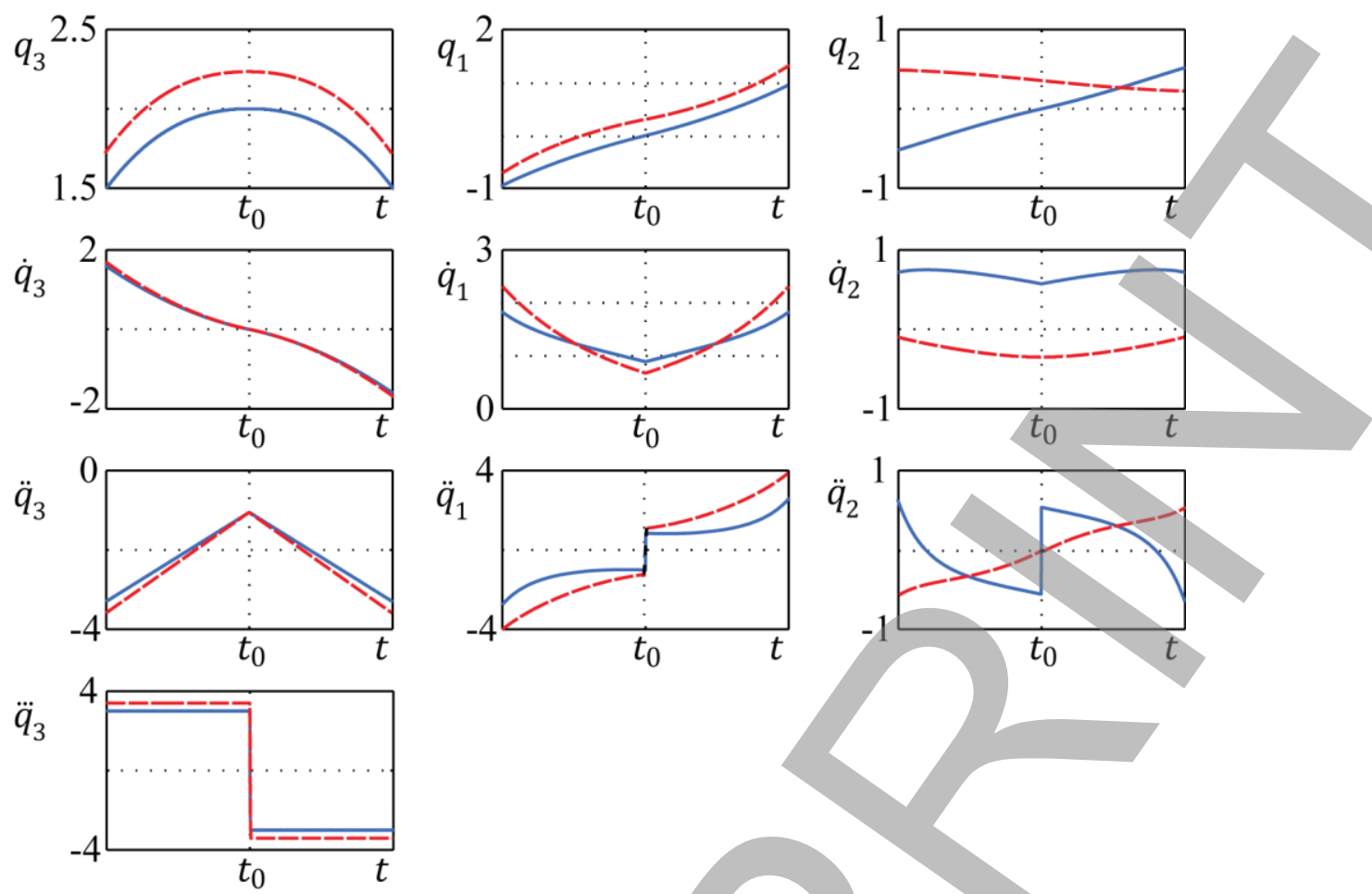

Figure 7: Motion law of $q_{3}$ with $\ddot{q}_{3}\left(t_{0}\right) \neq 0$ and $\dddot{q}_{3}\left(t_{0}\right) \neq 0$ for the four linkage (dashed line) and slider-crank (solid line) mechanisms. Units in SI.

\section{Experimental results}

A test bed was built to check the usefulness of the proposed method. As shown in Figure 8, it is made up by a planar four linkage mechanism and two DC motors. The first motor M1 commands, through a planetary gear reducer, the movement of the mechanism described by the dependent coordinate $q_{1}$ of the crank. This motor is connected to an external power supply and its motion is recorded using an incremental encoder. The second motor M2 acts as a brake through another planetary gear reducer and is connected to a secondary external power supply. The effect of the break is required to preload the mechanism and, hence, reduce the unwanted consequences of the clearance in both, the joints and the gear reducers. In order to know the time evolution of the angular velocity of the independent coordinate $q_{3}$, a gyroscope is attached to the rocker. The main characteristics of the test bed are shown in Table 2. 

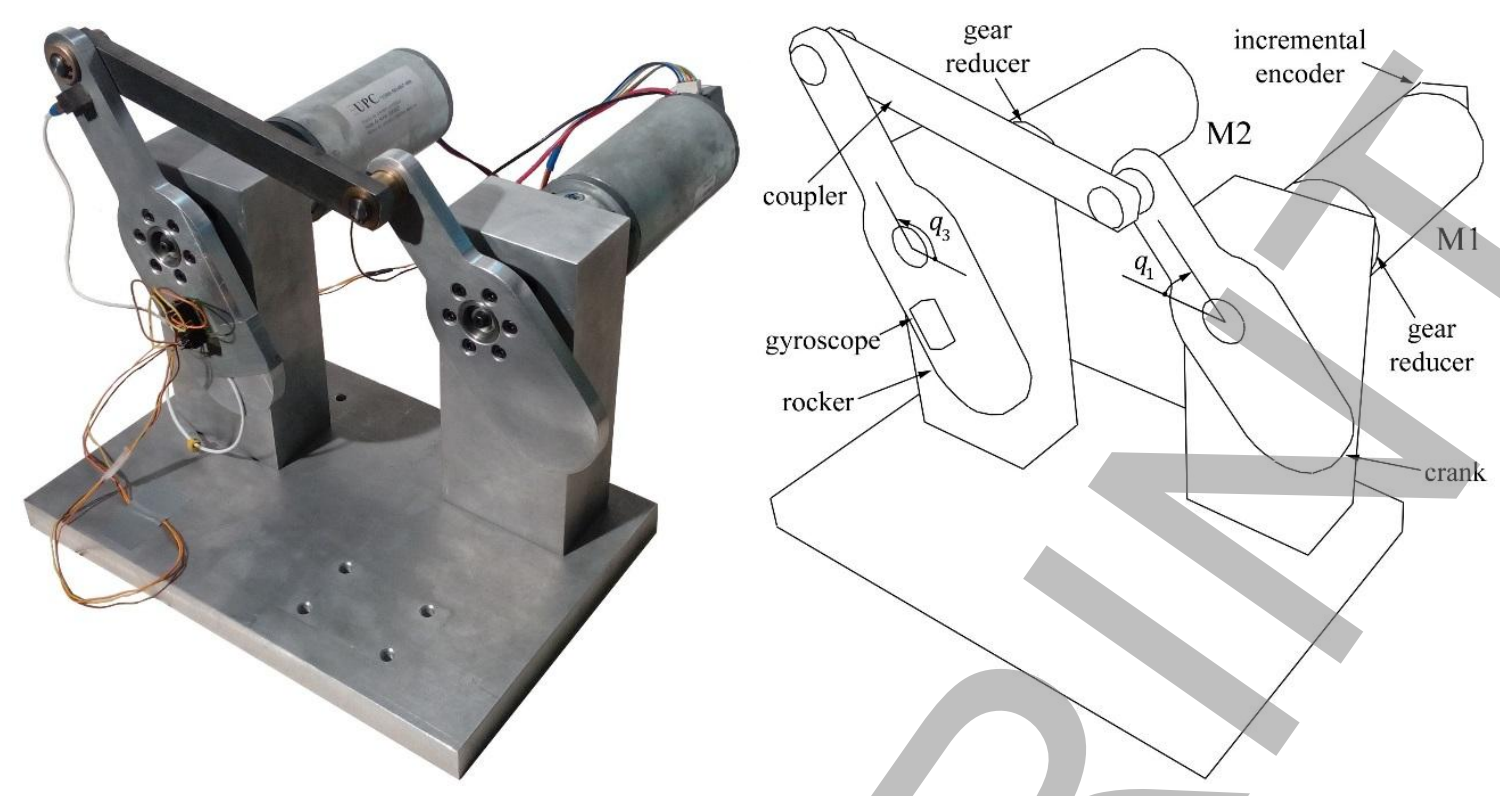

Figure 8: Test bed with a four linkage mechanism and two DC motors in any configuration a) and its scheme b).

\begin{tabular}{|c|c|}
\hline Parameter / device & Value $/$ specification \\
\hline$l_{1}$ & $0.075 \mathrm{~m}$ \\
\hline$l_{2}$ & $0.175 \mathrm{~m}$ \\
\hline$l_{3}$ & $0.100 \mathrm{~m}$ \\
\hline$l_{4}$ & $0.175 \mathrm{~m}$ \\
\hline Motor M1 and brake M2 & Permanent magnet DC brushed \\
\hline Gear reducer & Planetary with 20,25 ratio \\
\hline Encoder & Rotary, 500 cycles per revolution \\
\hline Gyroscope & ADXRS300, $\left.\pm 300^{\circ} / \mathrm{s}, 5 \mathrm{mV} /(\%) \mathrm{s}\right)$ \\
& Low pass filter RC, cutoff freq. at $40 \mathrm{~Hz}$ \\
\hline Power supply & DC $24 \mathrm{~V}, 5 \mathrm{~A}$ \\
\hline DAQ card & NI PCI- $6036 \mathrm{E}$ \\
\hline Gaussian FIR filter & Order 10, cutoff freq. at $50 \mathrm{~Hz}$ \\
\hline
\end{tabular}

Table 2: Test bed parameters and specifications. Nomenclature according to Figure 2. 


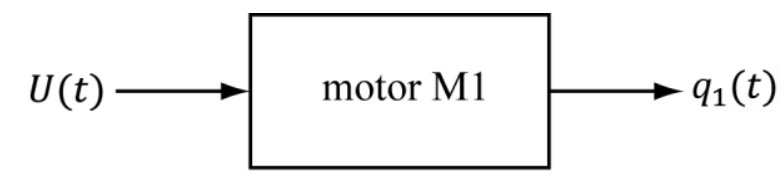

a)

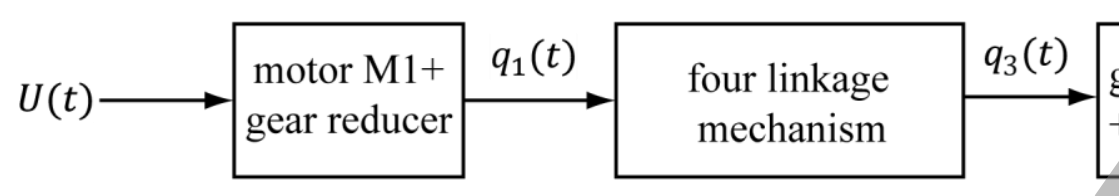

gear reducer
+ brake M2

b)

Figure 9: Block diagrams of the first a) and second experiments b).

In order to validate the method exposed in the previous sections, two experiments are carried out. The first one checks the feasibility of performing an acceleration step function as a command signal using conventional electrical actuators. For this reason, the motor M1 is connected, without any load, to a power supply in an open loop scheme according to Figure 9 a). The timedomain response of the motor is tested against a voltage step input $U(t)$ while the shaft orientation is recorded using the incremental encoder (Figure 10 a)). In order to obtain the angular velocity and acceleration of M1, this signal is differentiated twice (Figure $10 \mathrm{~b}$ ) and c), respectively). To reduce the level of the noise introduced by the derivative calculation, the acceleration $\ddot{q}_{1}$ has been filtered by the FIR Gaussian window specified in Table 2 . 


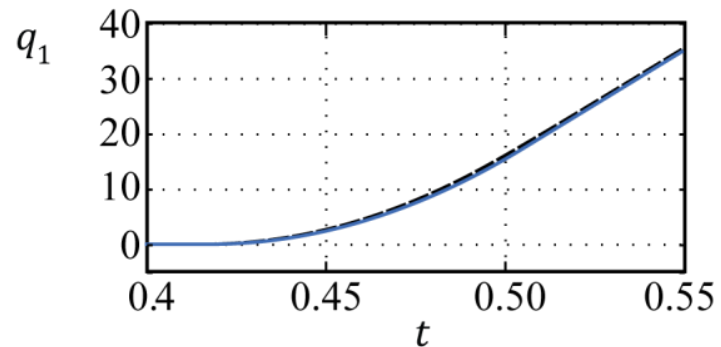

a)

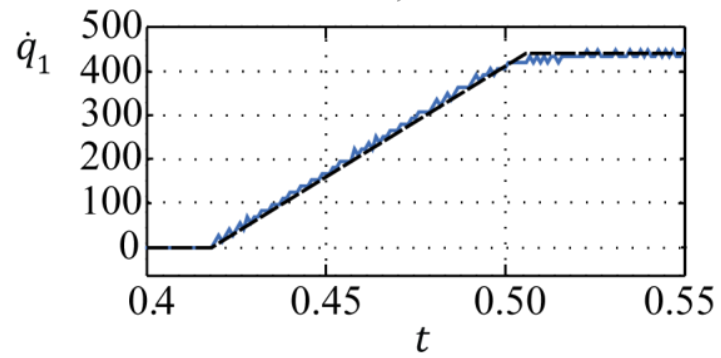

b)

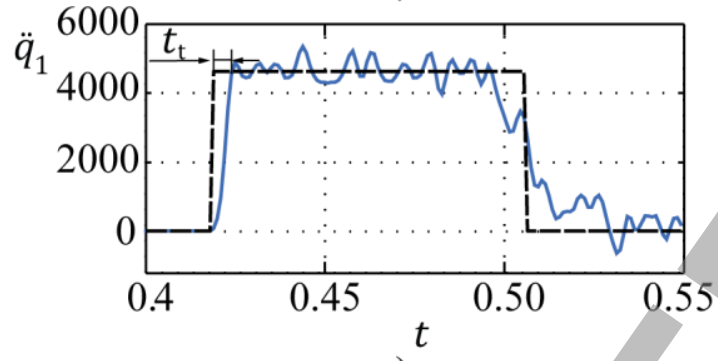

c)

Figure 10: DC motor response to a voltage step function, theoretical (dashed line) and experimental (solid line). Units in SI.

According to Figure 10, the delay $t_{\mathrm{t}}$ on the motor's response with respect to the reference signal is around $5 \mathrm{~ms}$. Thus, according to usual frequency ranges of mechanical systems, it can be sustained that conventional electrical actuators are able to reproduce an input command with continuity close to $\mathcal{C}^{1}$, as it is stated in section 3 .

The second experiment is intended to verify the continuity conditions exposed in section 5 . As in the former case, the set up follows an open loop scheme, where the input is the voltage $U(t)$ of the motor M1 (Figure $9 \mathrm{~b}$ )). For this purpose, and in order to be as accurate as possible with an ideal step function $\ddot{q}_{1}$, a voltage step is used to command the motor M1 when the four linkage mechanism is found at rest at the dead point configuration shown in Figure 11. In this specific configuration, $q_{1}=0.318 \mathrm{rad}$ and $q_{3}=2.246 \mathrm{rad}$. The results of this experiment are presented in Figure 12. As in the former experiment, $\ddot{q}_{1}$ has been obtained differentiating twice the signal from the incremental encoder. On the other hand, $\ddot{q}_{3}$ and $\dddot{q}_{3}$ have been obtained differentiating one and two times, respectively, the signal from the gyroscope $\dot{q}_{3}$. To reduce the noise, the signals, $\ddot{q}_{1}, \ddot{q}_{3}$ and $\dddot{q}_{3}$ have been filtered using the FIR Gaussian window (Table 2). The results present a small time delay, mainly caused by the low pass filter embedded in the gyroscope hardware and the digital Gaussian filter mentioned above. To better compare the goodness of the experiment, these delays have been removed to depict the graphical results. 

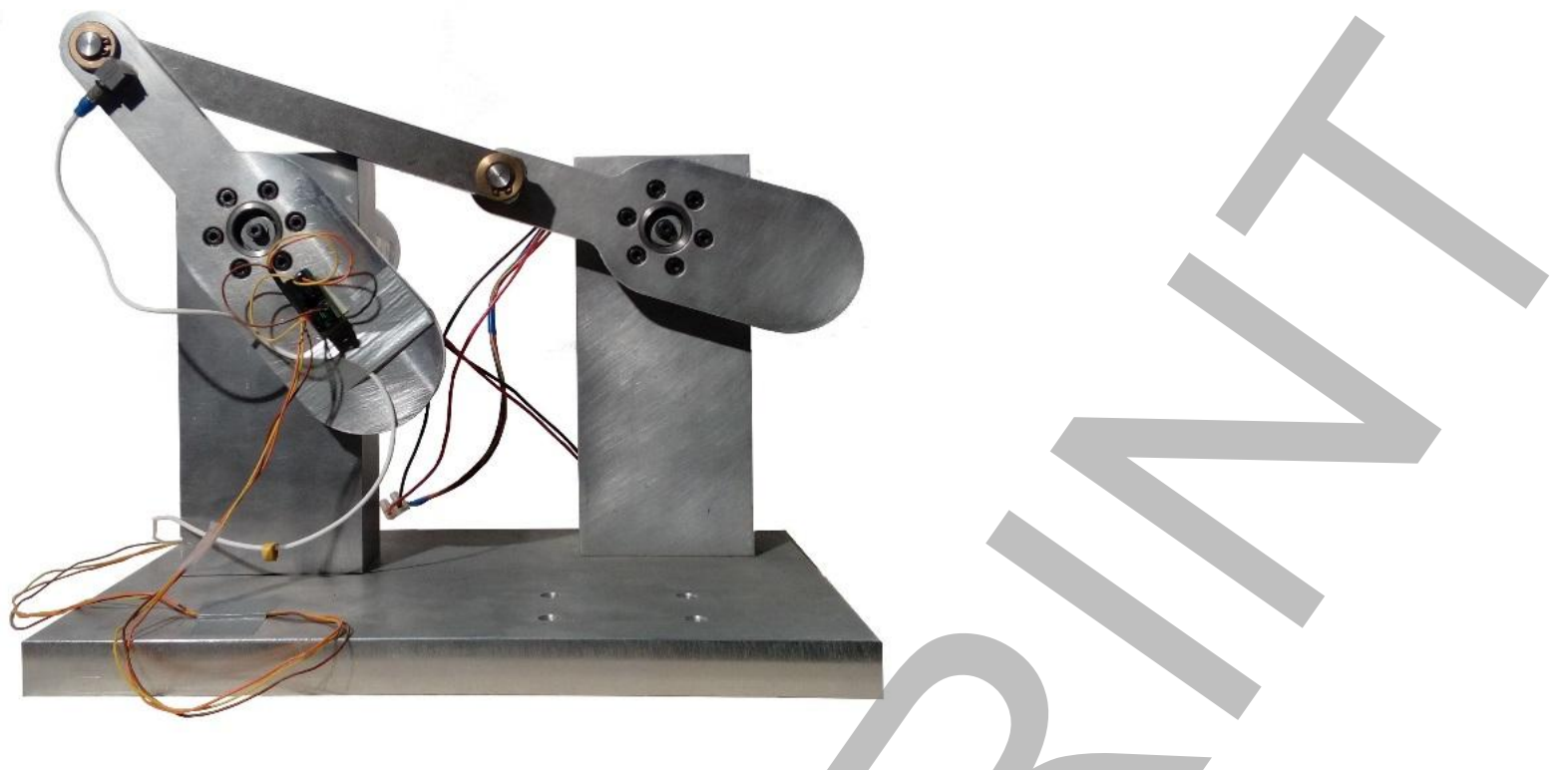

Figure 11: Four linkage mechanism at a dead point configuration for the independent coordinate $q_{3}$.

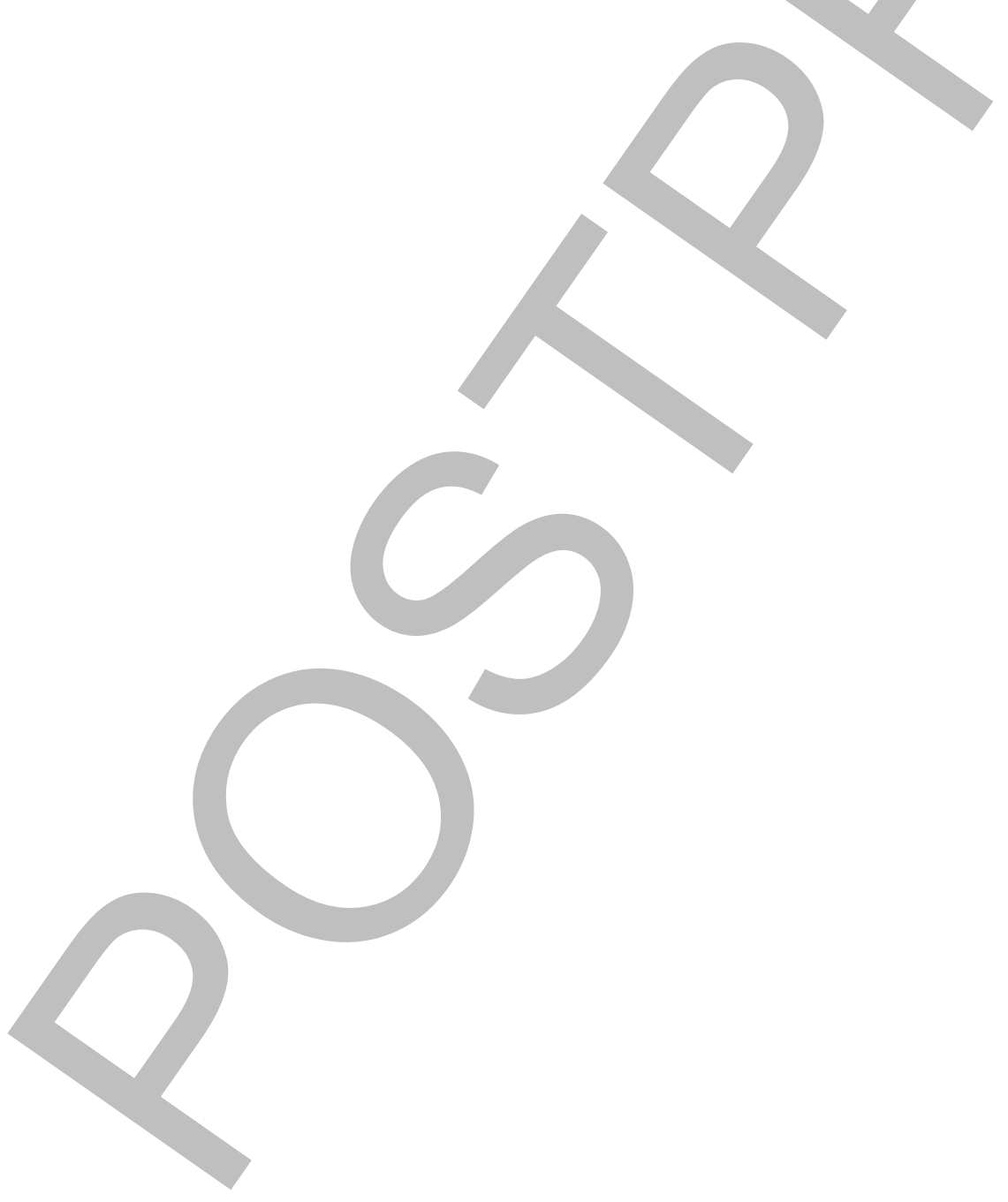



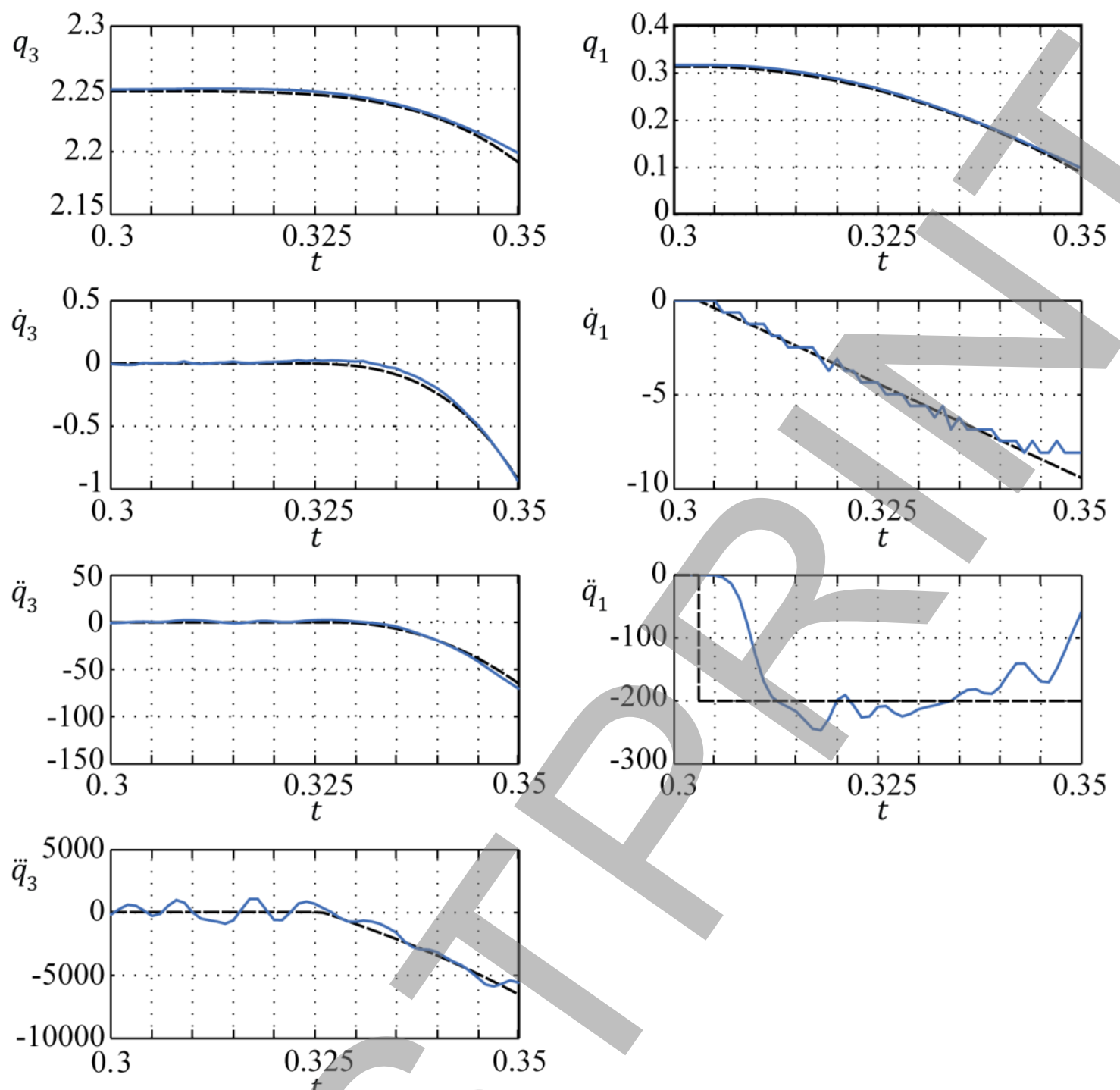

Figure 12: Motion law of the coordinates $q_{1}$ and $q_{3}$ during the experiment (solid line) and the theoretical curves (dashed line). Units in SI.

As it can be seen in Figure 12, a negative step function $\ddot{q}_{1}$ of $200 \mathrm{rad} / \mathrm{s}^{2}$ is obtained when the motor M1 is commanded with a step voltage, while the mechanism is at rest at the dead point configuration. The test bed is able to hold this acceleration during the first $35-40 \mathrm{~ms}$. Within this range, experimental results are very consistent with the theoretical simulation despite the limitations of the signal to noise ratios of the sensors, that become a drawback mainly in the high order derivatives $\ddot{q}_{1}$ and $\dddot{q}_{3}$.

\section{Conclusions}

The continuity $\mathcal{C}^{n}$ required by a motion law that describes the time-evolution of the independent coordinate of a mechanism when it presents dead points during its motion has been introduced in this paper. This analysis has been carried out differentiating and applying l'Hôpital's rule to the 
system of constraint equations $\boldsymbol{\phi}(\boldsymbol{q})$ of the mechanism and splitting the terms related with the dependent and independent generalized coordinates. This study also considered the feasibility of the time-domain profile to be reproduced with conventional electrical actuators (i.e. AC/DC motors, linear actuators, etc.). According to the study done in section 3 and the first experiment shown in section 6 , conventional electrical actuators can perform an input command that is, at least, $\mathcal{C}^{1}$. Regarding the continuity of the motion law of the independent coordinate $q^{\mathrm{i}}$ at a dead point configuration, it must be minimum $\mathcal{C}^{2}$ when $\ddot{q}^{\mathrm{i}} \neq 0$ and $\mathcal{C}^{3}$ when $\ddot{q}^{\mathrm{i}}=0$. In this last case $\left(\ddot{q}^{\mathrm{i}}=0\right)$, the value of the jerk $\dddot{q}^{\mathrm{i}}$ must necessarily also be null. Finally, the two simulations presented in section 5 and the experiment carried out in section 6 reinforce the generality and the goodness of the method.

\section{References}

[1] E. Kilic, C.U. Dogruer, M. Dolen and A.B. Koku, Position estimation for timing belt drives of precision machinery using structured neural networks, Mechanical Systems and Signal Processing 29 (2012) 343-361.

[2] J. Wang, G. He, J. Zhang, Y. Zhao and Y. Yao, Nonlinear dynamics analysis of the spur gear system for railway locomotive, Mechanical Systems and Signal Processing 85 (2017) 41-55.

[3] C. Bennett, J.F. Dunne, S. Trimby and D. Richardson, Engine cylinder pressure reconstruction using crank kinematics and recurrently-trained neural networks, Mechanical Systems and Signal Processing 85 (2017) 126-145.

[4] D.E. Whitney, Resolved motion rate control of manipulators and human prostheses, IEEE Transactions on man-machine systems 10 (1969) 47-53.

[5] A. Balestrino, G. De Maria and L. Sciavicco, Robust control of robotic manipulators, Proceedings of the 9th IFAC World Congress 5 (1984) 2435-2440.

[6] W.A. Wolovich and H. Elliot, A computational technique for inverse kinematics, IEEE Conference on Decision and Control (1984) 1359-1363.

[7] S.R. Buss, Introduction to Inverse Kinematics with Jacobian Transpose, Pseudoinverse and Damped Least Squares methods, IEEE Journal of Robotics and Automation (2004).

[8] Y. Nakamura and H. Hanafusa, Inverse kinematic solution with singularity robustness for robot manipulator control, ASME Journal of dynamic systems, measurement and control 108 (1986) 163-171.

[9] C.W. Wampler, Manipulator inverse kinematic solutions based on vector formulations and damped least-squares methods, IEEE Transactions on Systems, Man and Cybernetics 16 (1986) 93-101. 
[10] A.A. Maciejewski and C.A. Klein, Numerical Filtering for the Operation of Robotic Manipulators through Kinematically singular configurations, Journal of Robotic Systems 5 (1988) 527-552.

[11] S. Chiaverini., O. Egeland and K. Kanestrom, Achieving user-defined accuracy with damped least-squares inverse kinematic", Advanced Robotics 1 (1991) 672-677.

[12] A.S. Deo and I.D. Walker, Robot Subtask Performance with Singularity Robustness using Optimal Damped Least-Squares, IEEE International Conference on Robotics Automation 3 (1992) 434-441.

[13] S. Chiaverini, Estimate of the two smallest singular values of the jacobian matrix: applications to damped least-squares inverse kinematics, Journal of Robotic Systems 10 (1993) 991-1008.

[14] B. Siciliano and O. Egeland, Review of damped least-sauqres inverse kinematics with experiments on an industrial robot manipulator, IEEE Transactions on Control Systems Technology 2 (1994) 123-134.

[15] R. Buss and Jin-Su Kim, Selectively damped least squares for inverse kinematics, Journal of Graphics Tools 10 (2004) 37-49.

[16] J. Xiang, C. Zhong and W. Wei, General-weighted least-norm control for redundant manipulators, IEEE Transactions on Robotics 26 (2010) 660-669.

[17] T. Sugihara, Solvability-Unconcerned Inverse Kinematics by the Levenberg-Marquardt Method, IEEE Transactions on Robotics 27 (2011) 984-991.

[18] D.N. Nenchev, Tracking Manipulator trajectories with ordinary singularities: a null spacebased Approach, The International Journal of Robotics Research 14 (1995) 399-404, 1995.

[19] D.N. Nenchev and M. Uchiyama, Singularity-Consistent path tracking: A null space based approach, IEEE International Conference on Robotics and Automation 3 (1995) 2482-2489.

[20] J. Kieffer, Manipulator inverse kinematics for untimed end-effector trajectories with ordinary singularities, International Journal of Robotics Research 11 (1992) 225-237.

[21] D.N. Nenchev, Y. Tsumaki,, M. Uchiyama, V. Senft and G. Hirzinger, Two approaches to singularity consistent motion of nonredundant robotic mechanisms, IEEE International Conference on Robotics and Automation 2 (1996) 1883-1890.

[22] K. Tchoń and I. Dulęba, On inverting singular kinematics and geodesic trajectory generation for robot manipulators, Journal of Intelligent and Robotic Systems 8 (1993) 325-359.

[23] V. Senft and G. Hirzinger, Redundant motions of non-redundant robots - a new approach to singularity treatment, IEEE International Conference on Robotics and Automation (1995) 15531558. 
[24] S.F.M. Assal, K. Watanabe and K. Izumi, Neural Network-Based Kinematic Inversion of Industrial Redundant Robots Using Cooperative Fuzzy Hint for the Joint Limits Avoidance, IEEE/ASME Trans. on Mechatronics 11 (2006) 593-603.

[25] A.T. Hasan, M.A.A. Isa, H. Al-Assadi and A. Azlan Mat Isa, Neural Networks' Based Inverse Kinematics Solution for Serial Robot Manipulators Passing Through Singularities, INTECH Open Access Publisher (2011).

[26] A. Aristidou and J. Lasenby, FABRIK: a fast, iterative solver for the inverse kinematics problem, Graphical Models 73 (2011) 243-260.

[27] A.N. Pechev, Inverse kinematics without matrix inversion, IEEE International Conference on Robotics \& Automation (2008) 2005-2012.

[28] L.V. Vargas, A. C. Leite and R. R. Costa, Kinematic control of robot manipulators using filtered inverse, $21^{\text {st }}$ Mediterranean Conference on Control \& Automation (2013) 27-33.

[29] S. Cardona and D. Clos, Teoria de màquines, Edicions UPC, Barcelona, 2001.

[30] J.R. Brauer, Magnetic actuators and sensors, John Wiley \& Sons, 2006.

\section{Appendix A}

In section 2, it is stated that the numerator of Eq. (14) is zero when the mechanism is found at a dead point configuration for the independent coordinate.

$$
\operatorname{adj}\left(\phi_{q}^{\mathrm{d}}\right)\left[\phi_{q}^{\mathrm{i}} \ddot{q}^{\mathrm{i}}+\dot{\boldsymbol{\phi}}_{q} \dot{\boldsymbol{q}}\right]=0
$$

This can be proven by splitting the dependent and independent generalized coordinates in Eq. (A.1) according to Eqs. (6) and (7) and imposing $\dot{q}^{\mathrm{i}}=0$

$$
\operatorname{adj}\left(\boldsymbol{\phi}_{q}^{\mathrm{d}}\right)\left[\boldsymbol{\phi}_{q}^{\mathrm{i}} \ddot{q}^{\mathrm{i}}+\dot{\boldsymbol{\phi}}_{q}^{\mathrm{i}} \dot{q}^{\mathrm{i}}+\dot{\boldsymbol{\phi}}_{q}^{\mathrm{d}} \dot{\boldsymbol{q}}^{\mathrm{d}}\right]=\operatorname{adj}\left(\boldsymbol{\phi}_{q}^{\mathrm{d}}\right)\left[\boldsymbol{\phi}_{q}^{\mathrm{i}} \ddot{q}^{\mathrm{i}}+\dot{\boldsymbol{\phi}}_{q}^{\mathrm{d}} \dot{\boldsymbol{q}}^{\mathrm{d}}\right]=0
$$

Substituting now Eq. (20) in Eq. (A.2)

$$
\operatorname{adj}\left(\boldsymbol{\phi}_{q}^{\mathrm{d}}\right) \boldsymbol{\phi}_{q}^{\mathrm{i}}-\frac{\operatorname{adj}\left(\boldsymbol{\phi}_{q}^{\mathrm{d}}\right) \dot{\boldsymbol{\phi}}_{q}^{\mathrm{d}} \operatorname{adj}\left(\boldsymbol{\phi}_{q}^{\mathrm{d}}\right)}{\frac{\mathrm{d}}{\mathrm{d} t}\left(\operatorname{det}\left(\boldsymbol{\phi}_{q}^{\mathrm{d}}\right)\right)} \boldsymbol{\phi}_{q}^{\mathrm{i}}=0
$$


According to Jacobi's formula, Eq. (A.4) can also be written as

$$
\operatorname{adj}\left(\boldsymbol{\phi}_{q}^{\mathrm{d}}\right) \boldsymbol{\phi}_{q}^{\mathrm{i}}-\frac{\operatorname{adj}\left(\boldsymbol{\phi}_{q}^{\mathrm{d}}\right) \dot{\boldsymbol{\phi}}_{q}^{\mathrm{d}} \operatorname{adj}\left(\boldsymbol{\phi}_{q}^{\mathrm{d}}\right)}{\operatorname{tr}\left(\operatorname{adj}\left(\boldsymbol{\phi}_{q}^{\mathrm{d}}\right) \dot{\boldsymbol{\phi}}_{q}^{\mathrm{d}}\right)} \boldsymbol{\phi}_{q}^{\mathrm{i}}=0
$$

where $\operatorname{tr}\{\cdot\}$ is the trace of the matrix between brackets. As the determinant of the matrix $\boldsymbol{\phi}_{q}^{\mathrm{d}}$ is zero when the mechanism is found at a dead point, at least one of the rows is a lineal combination of the others $n-1$ equations. Therefore, without loss of generality, $\boldsymbol{\phi}_{q}^{\mathrm{d}}$ can be written as

$$
\boldsymbol{\phi}_{q}^{\mathrm{d}}=\left[\begin{array}{cccc}
h_{1,1} & h_{1,2} & \cdots & h_{1, n} \\
h_{2,1} & h_{2,2} & \cdots & h_{2, n} \\
\vdots & \vdots & \vdots & \vdots \\
h_{n-1,1} & h_{n-1,2} & \cdots & h_{n-1, n} \\
0 & 0 & \cdots & 0
\end{array}\right]
$$

Thus, the adjoin matrix of $\boldsymbol{\phi}_{q}^{\mathrm{d}}$ is

$$
\operatorname{adj}\left(\phi_{q}^{\mathrm{d}}\right)=\left[\begin{array}{cccc}
0 & \cdots & 0 & a_{1, n} \\
0 & \cdots & 0 & a_{2, n} \\
\vdots & \vdots & \vdots & \vdots \\
0 & \cdots & 0 & a_{n n}
\end{array}\right]
$$

Matrices $\boldsymbol{\phi}_{q}^{\mathrm{i}}$ and $\dot{\boldsymbol{\phi}}_{q}^{\mathrm{d}}$ can generically be written as

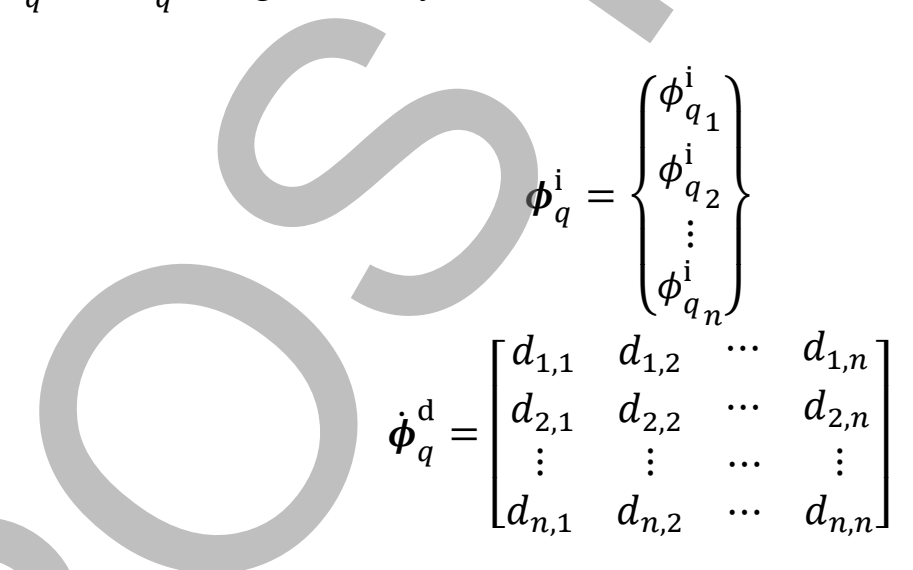

Substituting now Eqs. (A.7) and (A.8) in Eq. (A.5) 


$$
\left\{\begin{array}{c}
a_{1, n} \phi_{q_{n}}^{\mathrm{i}} \\
a_{2, n} \phi_{q_{n}}^{\mathrm{i}} \\
\vdots \\
a_{n, n} \phi_{q_{n}}^{\mathrm{i}}
\end{array}\right\}-\frac{\left[\begin{array}{cccc}
0 & \cdots & 0 & a_{1, n} \sum_{i=1}^{n} a_{i, n} d_{n, i} \\
0 & \cdots & 0 & a_{2, n} \sum_{i=1}^{n} a_{i, n} d_{n, i} \\
\vdots & \cdots & \vdots & \vdots \\
0 & \cdots & 0 & a_{n, n} \sum_{i=1}^{n} a_{i, n} d_{n, i}
\end{array}\right]}{\sum_{i=1}^{n} a_{i, n} d_{n, i}}\left\{\begin{array}{c}
\phi_{q_{1}}^{\mathrm{i}} \\
\phi_{q_{2}}^{\mathrm{i}} \\
\vdots \\
\phi_{q_{n}}^{\mathrm{i}}
\end{array}\right\}=0
$$

Rewriting Eq. (A.9)

$$
\left\{\begin{array}{c}
a_{1, n} \phi_{q_{n}}^{\mathrm{i}} \\
a_{2, n} \phi_{q_{n}}^{\mathrm{i}} \\
\vdots \\
a_{n, n} \phi_{q_{n}}^{\mathrm{i}}
\end{array}\right\}-\left\{\begin{array}{c}
a_{1, n} \phi_{q_{n}}^{\mathrm{i}} \\
a_{2, n} \phi_{q_{n}}^{\mathrm{i}} \\
\vdots \\
a_{n, n} \phi_{q_{n}}^{\mathrm{i}}
\end{array}\right\}=0
$$

Therefore, the numerator of Eq. (16) is equal to zero when the mechanism is located at a dead point.

\section{Appendix B}

In section 4, the expressions of the first and second derivatives of the matrix $\phi_{q}^{\mathrm{d}}$ with respect to the generalized coordinates $q^{\mathrm{d}}$ and $q^{\mathrm{i}}$ and their derivatives are needed. Thus, considering Eq. (6),

$$
\dot{\boldsymbol{\phi}}_{q}^{\mathrm{d}}=\frac{\mathrm{d}}{\mathrm{d} t}\left\{\boldsymbol{\phi}_{q}^{\mathrm{d}}\right\}=\left[\begin{array}{ccc}
\frac{\mathrm{d}}{\mathrm{d} t}\left(\frac{\partial \phi_{1}(\boldsymbol{q})}{\partial q_{1}}\right) & \cdots & \frac{\mathrm{d}}{\mathrm{d} t}\left(\frac{\partial \phi_{1}(\boldsymbol{q})}{\partial q_{n}}\right) \\
\vdots & \vdots \\
\frac{\mathrm{d}}{\mathrm{d} t}\left(\frac{\partial \phi_{n}(\boldsymbol{q})}{\partial q_{1}}\right) & \cdots & \frac{\mathrm{d}}{\mathrm{d} t}\left(\frac{\partial \phi_{n}(\boldsymbol{q})}{\partial q_{n}}\right)
\end{array}\right]
$$

According to the chain rule

$$
\dot{\boldsymbol{\phi}}_{q}^{\mathrm{d}}=\left[\begin{array}{ccc}
\frac{\partial}{\partial \boldsymbol{q}}\left(\frac{\partial \phi_{1}(\boldsymbol{q})}{\partial q_{1}}\right) \dot{\boldsymbol{q}} & \cdots & \frac{\partial}{\partial \boldsymbol{q}}\left(\frac{\partial \phi_{1}(\boldsymbol{q})}{\partial q_{n}}\right) \dot{\boldsymbol{q}} \\
\vdots & & \vdots \\
\frac{\partial}{\partial \boldsymbol{q}}\left(\frac{\partial \phi_{n}(\boldsymbol{q})}{\partial q_{1}}\right) \dot{\boldsymbol{q}} & \cdots & \frac{\partial}{\partial \boldsymbol{q}}\left(\frac{\partial \phi_{n}(\boldsymbol{q})}{\partial q_{n}}\right) \dot{\boldsymbol{q}}
\end{array}\right]
$$

Splitting the dependent and independent terms of Eq. (B.2) 


$$
\begin{aligned}
& \dot{\boldsymbol{\phi}}_{q}^{\mathrm{d}}=\left[\begin{array}{ccc}
\frac{\partial}{\partial \boldsymbol{q}^{\mathrm{d}}}\left(\frac{\partial \phi_{1}(\boldsymbol{q})}{\partial q_{1}}\right) \dot{\boldsymbol{q}}^{\mathrm{d}} & \cdots & \frac{\partial}{\partial \boldsymbol{q}^{\mathrm{d}}}\left(\frac{\partial \phi_{1}(\boldsymbol{q})}{\partial q_{n}}\right) \dot{\boldsymbol{q}}^{\mathrm{d}} \\
\vdots & & \vdots \\
\frac{\partial}{\partial \boldsymbol{q}^{\mathrm{d}}}\left(\frac{\partial \phi_{n}(\boldsymbol{q})}{\partial q_{1}}\right) \dot{\boldsymbol{q}}^{\mathrm{d}} & \cdots & \frac{\partial}{\partial \boldsymbol{q}^{\mathrm{d}}}\left(\frac{\partial \phi_{n}(\boldsymbol{q})}{\partial q_{n}}\right) \dot{\boldsymbol{q}}^{\mathrm{d}}
\end{array}\right]+ \\
& +\left[\begin{array}{ccc}
\frac{\partial}{\partial q^{\mathrm{i}}}\left(\frac{\partial \phi_{1}(\boldsymbol{q})}{\partial q_{1}}\right) \dot{q}^{\mathrm{i}} & \cdots & \frac{\partial}{\partial q^{\mathrm{i}}}\left(\frac{\partial \phi_{1}(\boldsymbol{q})}{\partial q_{n}}\right) \dot{q}^{\mathrm{i}} \\
\vdots & & \vdots \\
\frac{\partial}{\partial q^{\mathrm{i}}}\left(\frac{\partial \phi_{n}(\boldsymbol{q})}{\partial q_{1}}\right) \dot{q}^{\mathrm{i}} & \cdots & \frac{\partial}{\partial q^{\mathrm{i}}}\left(\frac{\partial \phi_{n}(\boldsymbol{q})}{\partial q_{n}}\right) \dot{q}^{\mathrm{i}}
\end{array}\right]
\end{aligned}
$$

Rewriting Eq. B.3

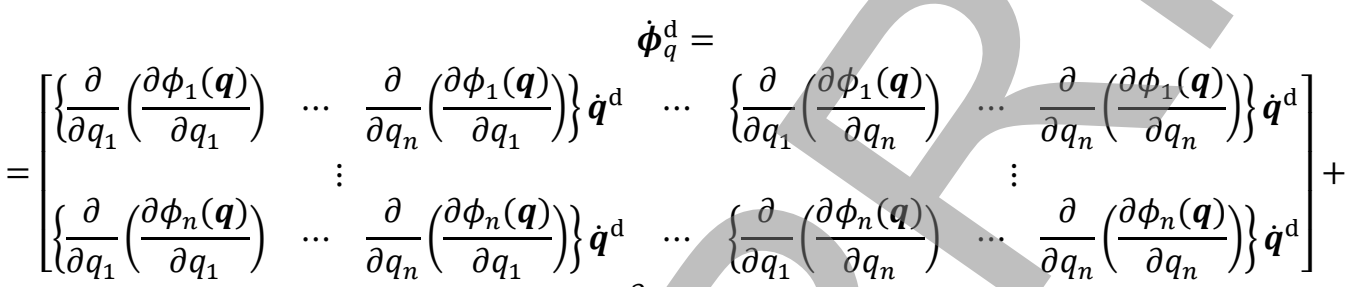

$$
\begin{aligned}
& +\frac{\partial}{\partial q^{\mathrm{i}}}\left(\boldsymbol{\phi}_{q}^{\mathrm{d}}\right) \dot{q}^{\mathrm{i}}
\end{aligned}
$$

Rearranging terms

$$
\dot{\boldsymbol{\phi}}_{q}^{\mathrm{d}}=\frac{\partial}{\partial q_{1}}\left(\boldsymbol{\phi}_{q}^{\mathrm{d}}\right) \dot{q}_{1}+\cdots+\frac{\partial}{\partial q_{n}}\left(\boldsymbol{\phi}_{q}^{\mathrm{d}}\right) \dot{q}_{n}+\frac{\partial}{\partial q^{\mathrm{i}}}\left(\boldsymbol{\phi}_{q}^{\mathrm{d}}\right) \dot{q}^{\mathrm{i}}
$$

Therefore,

$$
\dot{\boldsymbol{\phi}}_{q}^{\mathrm{d}}=\sum_{k=1}^{n}\left(\frac{\partial}{\partial q_{k}}\left(\boldsymbol{\phi}_{q}^{\mathrm{d}}\right) \dot{q}_{k}\right)+\frac{\partial}{\partial q^{\mathrm{i}}}\left(\boldsymbol{\phi}_{q}^{\mathrm{d}}\right) \dot{q}^{\mathrm{i}}
$$

Imposing $\dot{q}^{\mathrm{i}}=0$

$$
\dot{\boldsymbol{\phi}}_{q}^{\mathrm{d}}=\sum_{k=1}^{n}\left(\frac{\partial}{\partial q_{k}}\left(\boldsymbol{\phi}_{q}^{\mathrm{d}}\right) \dot{q}_{k}\right)
$$

For the calculation of the second order derivative of the matrix $\phi_{q}^{\text {d }}$, Eq. (B.6) is differentiated

$$
\ddot{\boldsymbol{\phi}}_{q}^{\mathrm{d}}=\sum_{l=1}^{n}\left(\frac{\mathrm{d}}{\mathrm{d} t}\left(\frac{\partial}{\partial q_{l}}\left(\boldsymbol{\phi}_{q}^{\mathrm{d}}\right)\right) \dot{q}_{l}+\frac{\partial}{\partial q_{l}}\left(\boldsymbol{\phi}_{q}^{\mathrm{d}}\right) \ddot{q}_{l}\right)+\frac{\mathrm{d}}{\mathrm{d} t}\left(\frac{\partial}{\partial q^{\mathrm{i}}}\left(\boldsymbol{\phi}_{q}^{\mathrm{d}}\right)\right) \dot{q}^{\mathrm{i}}+\frac{\partial}{\partial q^{\mathrm{i}}}\left(\boldsymbol{\phi}_{q}^{\mathrm{d}}\right) \ddot{q}^{\mathrm{i}}
$$


The term $\frac{\mathrm{d}}{\mathrm{d} t}\left(\frac{\partial}{\partial q_{l}}\left(\phi_{q}^{\mathrm{d}}\right)\right)$ can be substituted by Eq. (B.7), just replacing $\boldsymbol{\phi}_{q}^{\mathrm{d}}$ for $\frac{\partial}{\partial q_{l}}\left(\phi_{q}^{\mathrm{d}}\right)$. Then,

$$
\begin{gathered}
\ddot{\boldsymbol{\phi}}_{q}^{\mathrm{d}}=\sum_{l=1}^{n}\left(\sum_{k=1}^{n}\left(\frac{\partial}{\partial q_{k}}\left(\frac{\partial}{\partial q_{l}}\left(\boldsymbol{\phi}_{q}^{\mathrm{d}}\right)\right) \dot{q}_{k}\right) \dot{q}_{l}+\frac{\partial}{\partial q_{l}}\left(\boldsymbol{\phi}_{q}^{\mathrm{d}}\right) \ddot{q}_{l}\right)+\frac{\mathrm{d}}{\mathrm{d} t}\left(\frac{\partial}{\partial q^{\mathrm{i}}}\left(\boldsymbol{\phi}_{q}^{\mathrm{d}}\right)\right) \dot{q}^{\mathrm{i}}+ \\
+\frac{\partial}{\partial q^{\mathrm{i}}}\left(\boldsymbol{\phi}_{q}^{\mathrm{d}}\right) \ddot{q}^{\mathrm{i}}
\end{gathered}
$$

Considering finally $\dot{q}^{\mathrm{i}}=0$

$$
\ddot{\boldsymbol{\phi}}_{q}^{\mathrm{d}}=\sum_{l=1}^{n}\left(\sum_{k=1}^{n}\left(\frac{\partial}{\partial q_{k}}\left(\frac{\partial}{\partial q_{l}}\left(\boldsymbol{\phi}_{q}^{\mathrm{d}}\right)\right) \dot{q}_{k}\right) \dot{q}_{l}+\frac{\partial}{\partial q_{l}}\left(\boldsymbol{\phi}_{q}^{\mathrm{d}}\right) \ddot{q}_{l}\right)+\frac{\partial}{\partial q^{\mathrm{i}}}\left(\boldsymbol{\phi}_{q}^{\mathrm{d}}\right) \ddot{q}^{\mathrm{i}}
$$

